\title{
Framework for Multidisciplinary Integrated Modeling and Analysis of Space Telescopes
}

\author{
David W. Miller, Olivier L. de Weck and Gary E. Mosier
}

\begin{abstract}
This paper presents a comprehensive framework for integrated modeling, simulation and analysis of optical telescopes. This framework is called DOCS (Dynamics-Optics-Controls-Structures) and supports model development, model integration, analysis and multidisciplinary design optimization of this class of precision opto-mechanical systems. First the research background and literature in this young field is discussed. Next the structure and nominal process of an integrated modeling, simulation and analysis study for a generic optical telescope using the DOCS framework is discussed in detail. The major steps include subsystems modeling, model assembly, model reduction and conditioning, initial performance assessment, sensitivity analysis, uncertainty analysis, redesign, design optimization and isoperformance analysis. Such a comprehensive analysis is demonstrated for the NEXUS Space Telescope precursor mission. This mission was designed as a technology testbed for the Next Generation Space Telescope. The challenge is to achieve a very tight pointing accuracy with a sub-pixel line-of-sight (LOS) jitter budget and a root-mean-square (RMS) wavefront error smaller than $\lambda / 50$ despite the presence of electronic and mechanical disturbance sources. The framework suggested in this paper has the potential for becoming a general prescription for analyzing future, innovative telescope projects. Significant challenges remain in enabling fast simulations for large models, analytical sensitivity analysis for all sub-models, incorporation of slow-varying thermal or impulsive transient effects and the effective use of experimental results.
\end{abstract}

Keywords - Integrated Modeling, Telescopes, Nexus, Isoperformance, Multidisciplinary Design Optimization (MDO), Dynamics and Controls, Spacecraft Design, Optics, Sensitivity Analysis

\section{INTRODUCTION}

The next generation of space and ground based astronomical observatories such as the Next Generation Space Telescope (NGST), the Space Interferometry Mission (SIM) or the Terrestrial Planet Finder (TPF) will significantly surpass the present generation, for example the Hubble Space Telescope (HST), in terms of their sensitivity, angular resolution, spectral resolution and imaging stability [59], [35], [17]. The present work is motivated by the need to predict the dynamic behavior of these telescopes during the conceptual and preliminary design phases before substantial resources are committed towards a particular system architecture. Figure 1 shows the HST in the upper left corner and a number of pro-

David Miller, Associate Professor, Massachusetts Institute of Technology, Cambridge, MA 02139, U.S.A, millerd@mit.edu

Olivier de Weck, Assistant Professor, Massachusetts Institute of Technology, Cambridge, MA 02139, U.S.A, deweck@mit.edu

Gary Mosier, Integrated Modeling Lead for NGST, NASA Goddard Space Flight Center, gary.e.mosier.1@gsfc.nasa.gov posed successor spacecraft below. The science objectives for these missions are translated into functional requirements. These are further flowed down to engineering system requirements. It is the fulfillment of these engineering requirements, specifically relating to dynamics and controls, which constitute the notion of "performance" in the present paper.

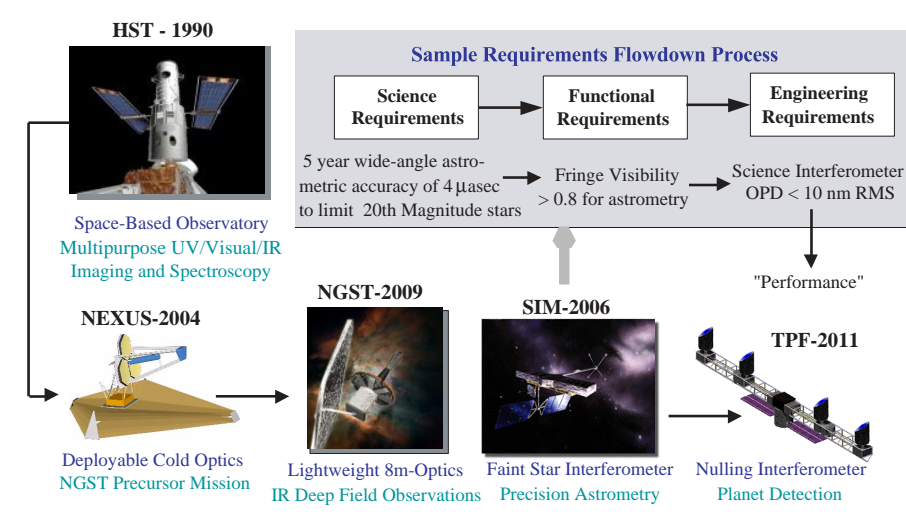

Fig. 1. Hubble Space Telescope and proposed successor missions as part of NASA's space science program. Sample requirements flowdown for SIM.

While the HST has performed admirably well over the last decade [17], it is essentially a multi-purpose instrument providing imaging and spectroscopy capabilities in the wavelength range $0.110-2.6[\mu \mathrm{m}]$, i.e. from ultraviolet (UV) to near-infrared (NIR). In order to achieve this large scope of science capabilities, a number of engineering compromises had to be made. The astronomical science community has realized that specialization is necessary such that the ambitious astrophysical research goals of the first half of the $21^{\text {st }}$ century (e.g. observation of proto-galaxies at high redshifts, $z$, direct IR detection of extra-solar earth-sized planets out to 15 parsecs) can be achieved [59]. Consequently a number of successor spacecraft have been proposed (Figure 1).

At first sight it appears impossible to attempt a unified engineering treatment of these various missions due to the large differences in their respective science objectives. Once these objectives have been broken down into tangible engineering requirements, however, the missions can be analyzed with a common set of tools. All missions require that electromagnetic radiation emanating from a science or guide source (e.g. star, proto-galaxy, extra-solar planet...) is collected by an aperture, compressed and 
redirected to an electronic detector (e.g. sprectrograph, CCD camera, fringe tracker). During this process it is paramount that the distortion of the wavefront (surface of common phase of light) inside the optical train be kept to a minimum, while the boresight axis of the observatory be held nearly fixed in inertial space.

For interferometers, additional requirements for the angular propagation of the wavefront (wavefront tilt-WFT), the pathlength the light travels in the different arms of the interferometer (optical pathlength difference-OPD) and the amount of overlap the interfering light beams experience at the detector (beamshear-BS) must be formulated. In order to ascertain that these telescopes will meet their stringent phasing and pointing requirements, the path from disturbance sources to the performance metrics of interest must be modeled in detail before construction, integration and testing. Additionally, for a number of light-weight deployable structures, pre-launch tests in a 1-g gravity field are not feasible. Hence, it is paramount that a preliminary design of the system is available, which can be used as a basis for a simulation model.

The science target observation mode is in quasi steadystate and is of particular importance. Other modes of interest can be transient such as the slewing and acquisition mode. Figure 2 shows a simplified block diagram of the main elements involved in a steady-state dynamics simulation. This is the reference problem setting considered in this paper. The premise is that a number of disturbance sources (reaction wheel assembly, cryocooler, guide star noise, etc.) are present during the science target observation mode as zero-mean random stochastic processes [6]. Their effect is captured with the help of state space shaping filters ${ }^{1}$, such that the input to the appended system dynamics is assumed to be a vector of unit-intensity white noises $d$, which are generally uncorrelated between disturbance sources. Reference input commands are designated as $r$. The simplest assumption is that the reference commands are zero, i.e. $r=0$, this, however, is not always the case.

The shaped disturbances $w$ are then propagated through the opto-structural plant dynamics, which include the structural dynamics of the spacecraft and the linear sensitivity optics matrices [63]. A compensator is often present in order to stabilize the observable rigid body modes (attitude control) and to improve the disturbance rejection or tracking capability (optical control). The sensor outputs $y$ and actuator inputs $u$ might also be subject to colored noise $n$. The goal of a disturbance analysis is to accurately predict the expected values of the performances $J_{z, i}$, where $i=1,2, \ldots, n_{z}$ and $n_{z}$ is the number of performance metrics. This has been previously developed and demonstrated by Gutierrez [25]. A summary of the disturbance, sensitivity and isoperformance analysis framework is contained in Appendix A. Outputs of the appended dynamics model are opto-scientific metrics of

${ }^{1}$ Sometimes these are referred to as pre-whitening filters.

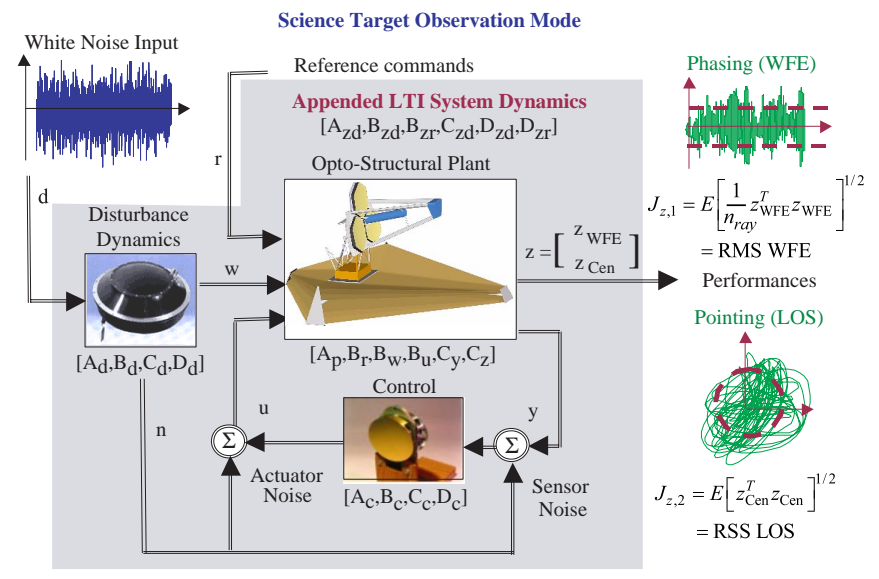

Fig. 2. Reference problem: Science target observation mode of a space telescope with pointing (RSS LOS) and phasing (RMMS WFE) performances

interest, $z$. The performances are typically expressed in terms of the root-mean-square (RMS) of the outputs. Alternatively we can combine channels in a RSS or RMMS metric, see Appendix A for details. Note that $n_{\text {ray }}$ is the number of light rays traced to compute the wavefront error (WFE). Other performance metrics could be the infinity norm $J_{z, i}=\left\|z_{i}\right\|_{\infty}$ or settling time $J_{z, i}=T s_{z, i}$ of a particular transient signal.

Another objective is to identify the "key" modal and/or physical parameters of the system that strongly drive the system performance. Sensitivity analysis has been previously identified [25] as a useful tool for examining the dependency of the predicted performance values $J_{z, i}$ on these "key" system parameters $p_{j}$, where $j=1,2, \ldots, n_{p}$ and $n_{p}$ is the number of parameters ${ }^{2}$. Some or all of the parameters might be subject to uncertainty. Oftentimes the number of parameters, $n_{p}$, for which a designer has to determine specific values exceeds the number of performance metrics, $n_{z}$, i.e. $n_{p}-n_{z} \geq 1$. The traditional approach is to first choose reasonable numbers for the system parameters $p_{j}$ and to predict the resulting performances $J_{z, i}$ (initial performance assessment). If all or some of the predicted performances do not initially meet the specified requirements $J_{z, r e q, i}$ for $i=1,2, \ldots, n_{z}$, including margins, a sensitivity analysis can provide partial derivatives $\partial J_{z, i} / \partial p_{j}$ which can be used to identify in which direction important parameters $p_{j}$ should be changed. This is intended to drive the system to a design point that satisfies all requirements, i.e. a condition where $J_{z, i} \leq J_{z, \text { req }, i}$ for all $i=1,2, \ldots, n_{z}$ is true. This process is called performance enhancement [25]. A $\pm \%$ uncertainty on the predicted performances, $\pm \triangle J_{z, i}$, can be computed based on known or assumed $\pm \%$ uncertainties of the parameters $p_{j}$. This is useful in establishing performance error bounds.

${ }^{2}$ It is assumed that these parameters are continuous over their interval $p_{j} \in\left[p_{L B, j} p_{U B, j}\right]$ 


\section{Literature ReVieW}

This section gives a short overview of the scientific literature which is relevant to the development and validation of integrated models for space and ground telescopes. The literature discussion begins with papers on the processes and tools used by systems engineers and designers during conceptual and preliminary design. The current stateof-the-art in performance assessment and enhancement of linear time-invariant systems is discussed along with initial work in the area of isoperformance methodology. The building blocks of integrated modeling of such systems are structural dynamics, classical and modern control theory, optical ray tracing as well as empirical and analytical modeling of various disturbance sources. In order to leverage these models and simulations in a multidisciplinary design optimization (MDO) context, issues of numerical conditioning, sensitivity analysis and uncertainty analysis cannot be ignored. Finally, past and presents efforts in laboratory testing and implementation on spaceflight missions are briefly discussed.

The conceptual and preliminary design phases are important times during a program in which various system architectures are analyzed and estimates are made of the top level and subsystem functional requirements. Additionally, initial budget allocations are made and enabling technologies are identified. The allocation of design requirements and resources (costs) and an assessment of risk during these early stages of a program is based on preliminary analyses using simplified models that try to capture the behavior of interest [12]. This was a major driver for the development of tools that allow quantitative analysis and design of these preliminary dynamics models early in a program. The kernel of the performance assessment (disturbance analysis), sensitivity and uncertainty analysis framework was established by Gutierrez [25]. The $\mathcal{H}_{2}$-type performances used here are defined in accordance with Zhou, Doyle and Glover [73].

The theory behind the performance assessment of linear dynamical systems is well-developed. A special case of the general performance assessment of a dynamical system is given when stochastic random noise processes are present. In this instance we speak of disturbance analysis and governing equations and methodologies are presented in random vibration textbooks such as those by Crandall [11] and Wirsching [71]. They characterize the response of systems driven by stochastic inputs in the time-domain (using autocorrelation functions) and equivalently in the frequency-domain (using power spectral density functions). The concept of a linear shaping or "pre-whitening" filter whose input is white noise and whose output is "colored" noise, presumably containing more disturbance energy in some frequency bands than in others, is covered by Brown and Hwang [6]. For the case of state-space systems driven by white noise, the output steady-state covariance matrix is known to be the solution of a Lyapunov equation [25].
The idea of holding a performance metric or value of an objective function constant and finding the corresponding contours has been previously explored by researchers in other areas. Gilheany [22] for example presented a methodology for optimally selecting dampers for multidegree of freedom systems [22]. In that particular work (Fig.5) the contours of equal values of the objective function $^{3}$ are found as a function of the damping coefficients $d_{11}$ and $d_{22}$. In the field of isoperformance methodology, work has been done by Kennedy, Jones and coworkers [41], [42], [40] on the need within the U.S. Department of Defense to improve systems performance through better integration of men and women into military systems (human factors engineering). They present the application of isoperformance analysis in military and aerospace systems design, by trading off equipment, training variables, and user characteristics. Once the level of operational performance is settled upon (e.g. "pilot will check out all aircraft flight systems within 30 seconds"), tradeoffs among equipment variables, adaptation, training, and individual predisposing factors can be made. A systematic approach to isoperformance in complex, dynamic opto-mechanical systems was developed by de Weck [14].

A field that has received a lot of attention in the last few years is integrated modeling. This encompasses research and efforts to simulate complex systems in a unified and multidisciplinary environment. Several important initiatives in this field, like NASA's Intelligent Synthesis Environment (ISE) described by Venneri, Malone and coworkers [70] were undertaken. Important contributions to integrated modeling were made by the Jet Propulsion Laboratory (JPL) with the creation of a MATLAB based finite element package and optical modeling software called IMOS (Integrated Modeling of Optical Systems) [37]. This code was developed to assist in the synthesis of initial models of optical instruments and to reduce the model creation, analysis and redesign cycle as described by Laskin and San Martin [44].

Structural dynamics fundamentals, in particular the single degree-of-freedom oscillator are treated by Craig [10]. More advanced concepts on the finite-element method are presented by Bathe [2] and Cook [9]. The structural dynamics and controls of large flexible spacecraft have been extensively studied by Junkins and Kim [38] as well as Crawley [12]. As will be seen later the stringent pointing and phasing requirements of optomechanical systems often require closed-loop attitude and optical control. Thus, actuators, sensors, and compensators must be included in the integrated model. Control textbooks by Van de Vegte [69] and Ogata [61] provide an overview of classical control design techniques, while those of Zhou et.al, [73] and Bélanger [3] emphasize modern control theory (state space based). Typically control sys-

\footnotetext{
${ }^{3}$ The objective function in reference $[22]$ is called ITSE $=$ integral of time multiplied by the sum of squares of displacements and velocities of the masses.
} 
tems are implemented on digital computers as described by Åström [1] and Franklin and Powell [20]. It is interesting to note that there still is a large discrepancy between theoretical modern control theory based on LQR, LQG and sensitivity weighted LQG, H-infinity and $\mu$-synthesis techniques and controllers that are actually implemented in space flight vehicles up to this day. Aerospace contractors, NASA and other space agencies still rely mainly on analog or digital implementations of classical control techniques such as PID and loop-shaping for attitude, thermal, optical and, among others, end effector control. This is likely due to an aversion towards risk and to the ease with which classical controllers are designed, understood, implemented and tested. Additionally the advantage of extensive flight heritage frequently offsets the potential performance benefits of modern controllers. This fundamental disparity was recognized by Mallory and Miller [49]. They proposed, developed and validated a MIMO controller tuning technique which begins with a simple local (often classical) baseline controller, which is then analyzed and iteratively tuned by opening up promising cross-channels and adjusting controller parameter settings based on a gradient search technique.

The fundamental work that allows the computation of optical linear sensitivity matrices and their incorporation into dynamics models is attributed to Redding and Breckenridge [63]. The linear sensitivity matrices allow computing optical metrics such as centroid position on the focal plane, wavefront error, wavefront tilt or beam shear as a function of linear and rotational displacements of the points where elements in the optical train (mirrors, beamsplitters, filter wheels etc.) are attached to the structure. The software program called MACOS ${ }^{4}$ (Modeling and Analysis for Controlled Optical Systems) [36] creates the sensitivity matrix based on a prescription of optical elements in the system and unit perturbations of the structural degrees-of-freedom. General recommended references for optics are by Born and Wolf [5] as well as Hecht [30]. Telescope optics in particular are described by Rutten and van Venrooij [64].

When considering a disturbance analysis it is important to enumerate and characterize all potential energy sources that might interfere with the opto-mechanical performance of the system. Eyerman and Shea [18] provide a very complete overview of spacecraft disturbances. Reaction wheel disturbances are often expected to be the dominant source and Bialke [4], Davis, Wilson, Jewell and Roden [13], Melody [52] as well as Masterson [51] have contributed to this field. Reaction wheel disturbance models are also included in this paper and an attempt is made to derive performance derivatives with respect to physical parameters of the wheels. Other likely disturbance sources are linear Sterling cryocoolers, attitude determination sensors, guide star noise in a CCD guider camera or $\mathrm{A} / \mathrm{D}, \mathrm{D} / \mathrm{A}$ conversion noise.

\footnotetext{
${ }^{4}$ formerly known as COMP
}

Once an integrated model has been assembled into a large appended state space model numerical conditioning becomes important, before it can be useful in a disturbance, sensitivity or isoperformance analysis. This is due to that fact that numerically ill-conditioned models are likely to produce inaccurate results in time simulations or when solving Riccati or Lyapunov equations. Additionally it is often true that the dynamic response of a complex opto-mechanical system is often dominated by a small subset of states or modes. Large computational savings can be obtained if this subset can be identified and isolated apriori via model conditioning and reduction. Creating a balanced state-space realization according to Moore [57] can not only improve the numerical conditioning properties of a model, but it also serves as a technique for identifying states that can be eliminated from the model due to low observability and controllability [73]. Additional techniques for balancing large state space models are presented by Laub and coworkers [45], [46]. For the case of systems dominated by lightly-damped modes, the method proposed by Gregory [23] is an efficient approach for ranking the importance of these modes. An effective pre-balancing technique was proposed by Mallory [49]. A comprehensive view of model quality management was taken by Uebelhart [68].

A sensitivity analysis provides useful information as to how dependent outputs of a model are with respect to modal or physical parameters of the model. A key aspect of the isoperformance methodology is the ability to compute exact analytical sensitivities, as opposed to inexact and inefficient finite difference based gradients, which are strongly affected by the chosen perturbation size. These are then assembled into a gradient vector or Jacobian matrix in the multivariable case. The isoperformance technique operates in the nullspace of the Jacobian. A good overview of linear algebra in this context, describing the nullspace and singular value decomposition of a matrix, is provided by Strang [67]. Analytical expressions for the sensitivities of performance metrics previously derived by Gutierrez [25] are used throughout this paper. A Lagrange multiplier approach was proposed by Jacques [33] to obtain analytical sensitivities of a system's outputs with respect to various parameters. The calculation of sensitivities requires mode shape and frequency derivatives, which fall under the category of eigenvalue and eigenvector derivatives. A good survey of various eigenderivative methods is provided by Murthy and Haftka [58]. When the parameters are element mass and stiffness properties of a finite-element model, these derivatives can be computed exactly using methods developed by Fox and Kapoor [19] and Nelson [60]. Practical implementation of these methods is done by Kenny [43], and this work is extended by Gutierrez [25]. Previous work by Hou and Koganti [31] in the context of integrated controls-structure design also makes extensive use of sensitivities.

Since not all parameters in a system are known to the 
same degree, parametric uncertainty represents an important element of risk. Uncertainty analysis of the performances (performance robustness) and stability margins (stability robustness) are the topic of publications by Yang [72], How [32], Skelton [66], Zhou et. al., [73] and the work by Campbell and Crawley [8]. An approximate method for predicting worst-case performance RMS values due to parametric uncertainties is that used by Bryson and Mills [7]. One particular method is the first-order approach that relates the covariance matrix of output quantities in terms of the covariance matrix of uncertain parameters and the sensitivity matrix of the outputs with respect to the parameters.

The multiobjective design optimization aspects of this work draw on previous research results in multidisciplinary design optimization. A fundamental book on the theory of multiobjective optimization was published by Sawaragi, Nakayama and Tanino [65]. An important application of multiobjective optimization is concurrent control/structure optimization. The objective is to develop structure and control designs simultaneously such that the overall system design has improved properties compared to a system design obtained through a traditional, sequential approach. This allows the same performance to be achieved with less control effort or less structural mass, for example. This philosophy is carried on in this paper by additionally including disturbance and optical design parameters. Solutions of these multidisciplinary optimizations are dependent on the type of objective functionals specified and the programming techniques employed. The method developed by Milman et. al., [56] does not seek the global optimal design, but rather generates a series of Pareto-optimal designs that can help identify the characteristics of better system designs. This work comes closest to the spirit followed in this paper. A good overview of structural and multidisciplinary optimization research is given in the volume "Structural Optimization: Status and Promise" edited by Kamat [39].

It is interesting to note that the research presented on dynamics and controls of actual precision spacecraft or laboratory testbeds is much less voluminous than the theoretical literature in the field. The integrated modeling process is demonstrated by Melody and Neat [53] on JPL's Micro-Precision Interferometer (MPI) testbed and is experimentally validated based on the comparison of predicted and measured closed-loop transfer functions. Optical pathlength control of a JPL Phase B interferometer testbed was presented by O'Neal and Spanos [62]. Another example is the work done at NASA Langley Research Center by Maghami [47]. The pointing performance of a large, laboratory testbed was successfully maintained while control effort was decreased. The genetic algorithm approach to evolutionary design of flexible structures developed by Masters was experimentally validated on a closed-loop, truss-like testbed [50]. Mallory [49] presents experiments of slewing, pointing and phasing control of the ORIGINS space telescope testbed.

Apart from the more generic literature in the field we can also find publications devoted to specific missions. Disturbance analysis results for space optical systems have been presented for SIM by Grogan and Gutierrez [24],[26], as well as by de Weck along with Miller, Uebelhart, Grogan and Basdogan [55]. Open loop and closed loop NGST results have been published by de Weck, Miller and Mosier [15]. A preliminary analysis of the dynamics of the Terrestrial Planet Finder mission was also prepared by Miller, de Weck and co-workers [54].

\section{DOCS ANALYSIS FRAMEWORK}

The DOCS (Dynamics-Optics-Controls-Structures) framework presented in this paper is a powerful tool set for the modeling and analysis of precision opto-mechanical systems. Its development spans over the last 10 years at MIT's Space Systems Laboratory and the former Space Engineering Research Center (SERC). Within the MATLAB environment a model of the spacecraft (or ground system) can be created, which simulates the dynamic behavior of the structure, the optical train, the control systems and the expected disturbance sources in an integrated fashion (Figure 3). The existing toolbox is compatible with IMOS (version 5.0) and MACOS ${ }^{5}$, MSC/NASTRAN as well as the packages DynaMod and ControlForge ${ }^{6}$.

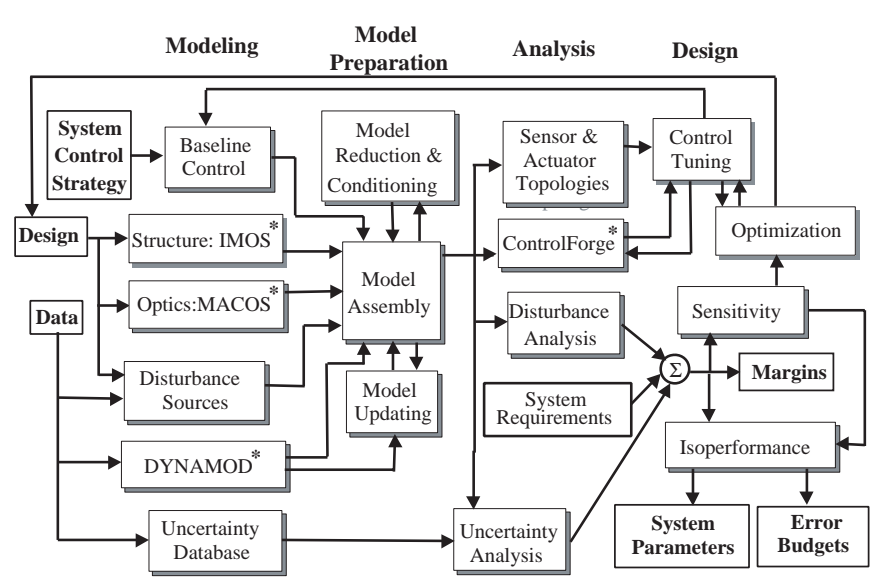

Fig. 3. DOCS-Framework block diagram. The modules marked with an asterisk are available commercially or through academic licensing.

The modules in DOCS can be subdivided into four categories, which correspond to the columns of the block diagram in Figure 3. The first column contains modules which are useful in the initial modeling of the dynamic system under investigation. This system can be a conceptual or preliminary design model of a scientific spacecraft, a spaceborne or ground based telescope or some other high

${ }^{5}$ A vailable for academic licensing from the Jet Propulsion Laboratory (JPL).

${ }^{6}$ Available commercially from Midé Technology Corporation. 
performance dynamic system. The initial design is the starting point for the creation of a structural model, which provides the stiffness and mass distribution of the system. This is typically done via the finite element method (FEM) and produces the natural frequencies, $\Omega$, and mode shapes, $\Phi$, which are used to obtain a state space representation of the structural dynamics. This can be done via commercial codes such as FEMAP and MSC/NASTRAN or with the help of IMOS. The corresponding optical model typically traces a large number of on-axis and off-axis rays through the system to obtain the linear sensitivity matrices. These matrices relate motions of the attachment points of optical elements to the optical performance metrics (wavefront error, optical path difference, wavefront tilt, beam shear etc.). DOCS has provisions for modeling a large variety of on-board and external disturbance sources, such as reaction wheel noise, cryocooler disturbances, thermal snap, $\mathrm{D} / \mathrm{A}$ and $\mathrm{A} / \mathrm{D}$ noise and various sensor noises. In some cases, if disturbance measurement data is available, these disturbance models are empirical and based on actual disturbance data from spacecraft operations or laboratory tests. The module DYNAMOD provides an alternative to finite element modeling, if physical measurements can be made on the system or subsystem. In that case model fitting techniques are used to obtain a MIMO measurement model which is a good fit to the experimentally determined transfer function matrix of the actual system (measurement model). Most high performance dynamic systems rely on feedback control systems to achieve the required performance levels. Thus, a baseline controller (compensator) must be synthesized based on a chosen system control strategy (e.g. local versus global control as in decoupled control loops for attitude and optical control, classical control versus modern control). Finally, it is likely that physical or modal parameters of the system model are uncertain. This uncertainty can be estimated apriori by consulting an uncertainty database of past model errors of similar systems, such as the one provided by Hasselman [27], [28], [29].

The second category of tools in the DOCS framework is dedicated to model preparation and conditioning. Model assembly is the process of aggregating the disciplinary sub-models into an overall, integrated model of the system dynamics. This assembled model will be open or closed-loop an can be mathematically represented in transfer matrix form $G_{z d}(j \omega)$, as an appended state space system, $S_{z d}$, or in system block diagram form (e.g. in Simulink). Typically the integrated model will initially be numerically ill-conditioned. A number of tools are provided to facilitate model "quality management". This includes verification of absolute and relative stability (margins), extraction of minimal representations, model balancing and reduction as well as model diagonalization. Model reduction is a critical step, since it reduces the model size leading to faster model evaluation, while maintaining the critical information. The recommended model conditioning in DOCS is a two step procedure with an initial conditioning of the opto-structural plant dynamics alone. The objective of this first step is to eliminate pole-zero cancellations and obtain a well conditioned plant model. The second conditioning and reduction step is more aggressive and is executed on the aggregated model, which contains the frequency weighting introduced by control and disturbance dynamics. Finally a number of software routines are emerging in DOCS which allow model updating of the plant model with measurement data.

After an integrated model has been created and numerically conditioned, the performances such as root-meansquare (RMS) values of opto-mechanical metrics (e.g. pathlength difference, pointing jitter, fringe visibility, nulldepth) can be predicted. This capability is provided by the disturbance analysis module, which appears in the third column of the DOCS block diagram. The disturbance analysis can be conducted in the time domain, frequency domain or as a Lyapunov analysis. The predicted performance values are compared with the requirements. The goal of the uncertainty analysis is to associate error bars with the predicted performance values, which are based on an uncertainty database resulting from past ground and flight experience. The actuator-sensor topology of the system can be analyzed numerically to ensure that the control system uses the actuator-sensor pairs that will ensure maximum disturbance rejection or tracking performance. This gives an indication if additional sensor-actuator channels should be included in the compensator beyond the ones in the baseline controller. The controller can also be analyzed and modified with ControlForge. This module is the product of control synthesis and analysis work on MACE and other experimental programs and allows implementation of modern control techniques such as sensitivity-weighted LQG.

An initial design will usually not be satisfactory without some amount of iteration. The exact analytical sensitivities of the performance with respect to modal or physical design parameters can be useful in this instance. These sensitivities are essential for conducting gradient-based optimization, redesign or uncertainty analyses. This capability is used in the model updating, ControlForge, uncertainty analysis, optimization, sensitivity and isoperformance modules shown in Figure 3. These activities are part of the "design" category of tools in DOCS. A control tuning engine was developed by Mallory [49] such that a parameterized controller is tweaked to provide improved performance with satisfactory stability margins for a fixed plant. An outer optimization loop is carried out during performance enhancement. Here insights from Controlled Structures Technology (CST) are levied such that a combination of performance enhancement steps (disturbance isolation, output isolation, plant redesign, low-authority control (LAC), high-authority control (HAC)) is implemented in the system design. Once a design has been found that meets all requirements with sufficient mar- 
gins, an isoperformance analysis can be conducted [14]. Treating the performance as an equality constraint, the expected error sources (error budgeting) or key design parameters (multiobjective design optimization) can be traded with respect to each other. This is useful in ensuring that the level of difficulty in implementing the design is similar for all subsystems. The framework has been successfully applied to conceptual designs of SIM, NGST, the Terrestrial Planet Finder (TPF) mission and NEXUS. Substantial contributions to the framework were made by Blaurock, Gutierrez [25], Jacques [34], Mallory [48], [49], Uebelhart [68], Masterson [51], de Weck [14] as well as Miller.

\section{NEXUS Space Telescope Study}

The purpose of the NEXUS spacecraft case study is to demonstrate the usefulness of the Dynamics-OpticsControls-Structures (DOCS) framework on a realistic conceptual design model of a high-performance spacecraft. A graphical representation of the launch and on-orbit configurations of NEXUS is shown in Figure 4.

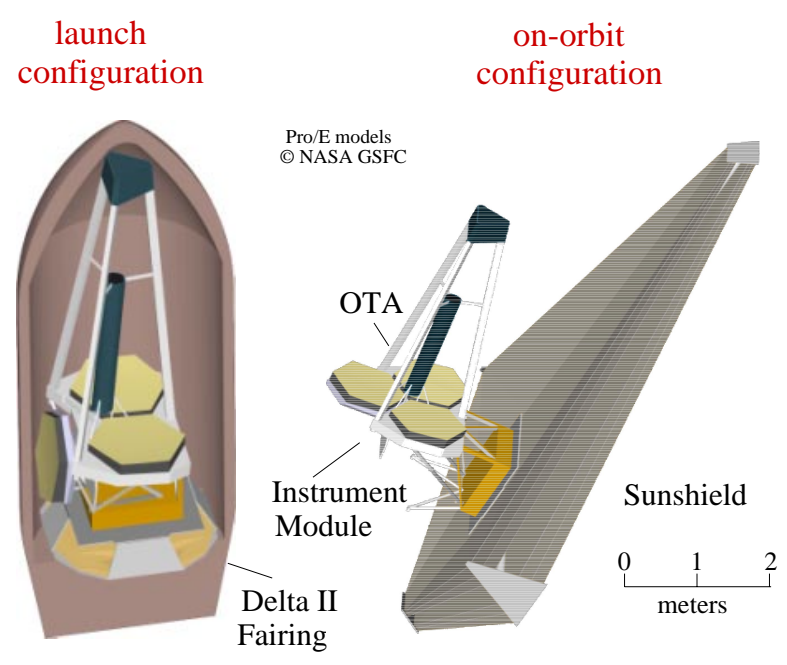

Fig. 4. NEXUS Spacecraft Concept

NEXUS was planned as a technology risk-reduction experiment in space and as a precursor to NGST. The NEXUS project was officially canceled in December 2000 as a part of the NGST rescoping exercise. It was nevertheless decided to use NEXUS for this case study, since the conceptual model is well developed and many lessons learned from previous NGST Yardstick models were incorporated.

NEXUS features a $2.8 \mathrm{~m}$ diameter primary mirror, consisting of three AMSD-sized primary mirror (PM) petals. Two of these are fixed and one is deployable as shown in Figure 4 on the left side. The total mass of the spacecraft is nominally $752.8[\mathrm{~kg}]$ at a cost of $\$ \mathrm{M} 105.88$ (FY00). The target orbit is L2 of the Sun/Earth system with a projected launch date of 2004. The optical telescope assembly (OTA) also features a 3-legged spider, which sup- ports the secondary mirror (SM). The instrument module contains the optics downstream of the tertiary mirror and the camera (detector). The sunshield is large, deployable and light-weight,thus accounting for the first flexible mode of the spacecraft structure around $0.2 \mathrm{~Hz}$.

\section{A. Integrated Modeling}

The integrated model for NEXUS contains a structural finite element model (FEM), see Figure 5. The model was initially created in FEMAP/NASTRAN and subsequently translated to IMOS [37]. The figure shows the important locations at which disturbance and control inputs enter as well as important output nodes for the ACS as well as the locations where optical elements are mounted.

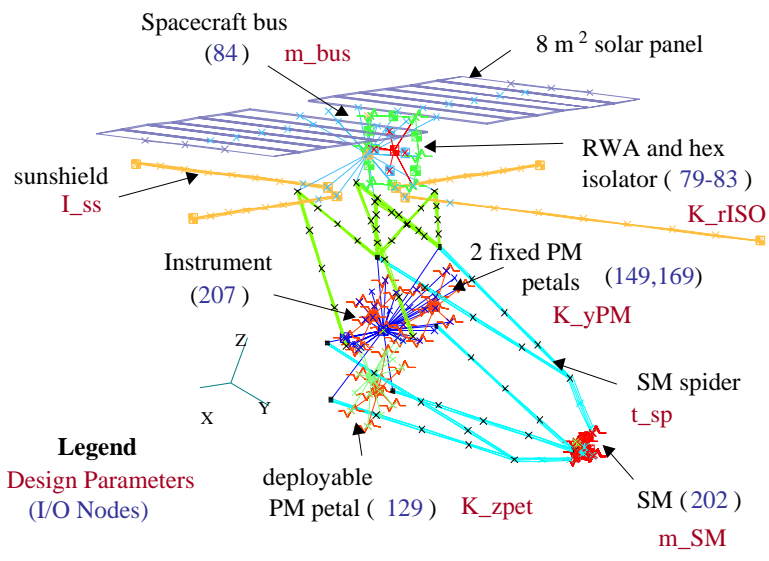

Fig. 5. NEXUS Finite Element Model. Important I/O grid points (nodes) and variable design parameters are shown.

The two performance metrics of interest are the rootmean-mean-square wavefront error, $J_{z, 1}=$ RMMS WFE, and the root-sum-square line-of-sight jitter, $J_{z, 2}=$ RSS LOS. The optical linear sensitivity matrices for these performance with respect to the translations and rotations of the optical elements were computed with MACOS. The appended dynamics of this system are shown in the block diagram of Figure 6.

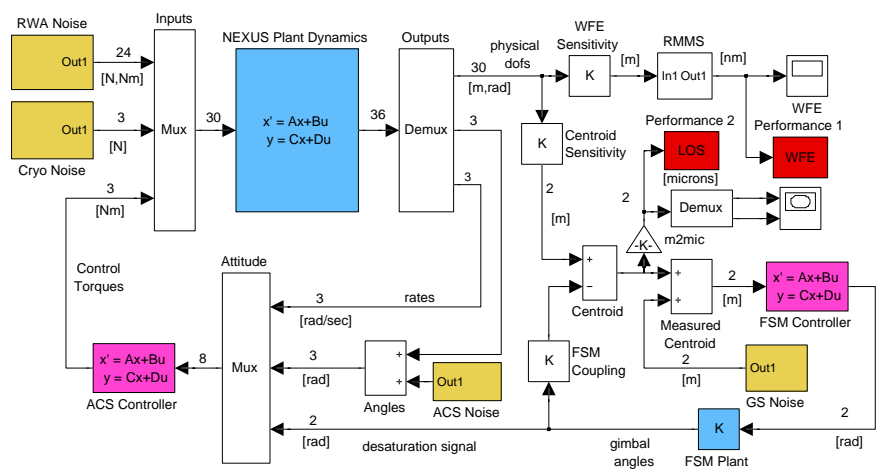

Fig. 6. NEXUS block diagram with 4 disturbance sources (RWA, Cryo, ACS noise, GS noise) and 2 performances (RMMS WFE, RSS LOS). Simulation implemented in Simulink as well as state space. 
There are four expected disturbance sources in the model $\left(n_{d}=4\right)$. The first is broadband reaction wheel noise, assuming a 4-wheel pyramid and uniform probability density on the wheel speed distribution, with an upper (operational) wheel speed $R_{u}$. The disturbance forces and torques are caused by static and dynamic imbalances, $U_{s}$ and $U_{d}$, as well as higher harmonics [51]. The second disturbance is due to a linear Sterling cryocooler at drive frequency $f_{c}$. This device is used to cool the IR detector and is installed in the instrument module. The third disturbance is attitude noise, which is based on rate gyro noise and star tracker noise measured on the Cassini mission (JPL). Finally there is guide star noise, which is very sensitive to the guider sampling rate, $T_{g s}$, and the guide star brightness, $M_{g s}$.

In summary the appended dynamics, $S_{z d}$, of this system contain 320 states $\left(n_{s}=320\right)$, two performance metrics $\left(n_{z}=2\right)$, four disturbance sources $\left(n_{d}=4\right)$ and 25 variable design parameters $\left(n_{p}=25\right)$. Table I summarizes the variable design parameters for the NEXUS case study.

TABLE I

NeXus Variable Design Parameters $p_{j}, j=1, \ldots, 25$.

\begin{tabular}{|c|c|c|c|}
\hline Symbol & $p_{o}$ & Description & Units \\
\hline & & \multicolumn{2}{|l|}{ disturbance parameters } \\
\hline$\overline{R_{u}}$ & 3000 & upper wheel speed & $\overline{[\mathrm{RPM}]}$ \\
\hline$U_{s}$ & 1.8 & static imbalance & {$[\mathrm{gcm}]$} \\
\hline$U_{d}$ & 60 & dynamic imbalance & {$\left[\mathrm{gcm}^{2}\right]$} \\
\hline$f_{c}$ & 30 & cryo drive frequency & {$[\mathrm{Hz}]$} \\
\hline$Q_{c}$ & 0.005 & cryo attenuation & {$[-]$} \\
\hline Tst & 20 & star tracker rate & {$[\mathrm{sec}]$} \\
\hline$S_{r g}$ & $3 \mathrm{e}^{-14}$ & gyro noise intensity & {$\left[\mathrm{rad}^{2} / \mathrm{s}\right]$} \\
\hline$S_{s t}$ & 2 & tracker one sigma & {$[\operatorname{arcsec}]$} \\
\hline$T_{g s}$ & 0.04 & GS integration time & {$[\mathrm{sec}]$} \\
\hline \multicolumn{4}{|c|}{ plant parameter } \\
\hline$m_{S M}$ & 2.49 & mass of secondary & {$[\mathrm{kg}]$} \\
\hline$K_{y P M}$ & $0.8 \mathrm{e} 6$ & PM bipod stiffness & {$[\mathrm{N} / \mathrm{m}]$} \\
\hline$K_{r I S O}$ & 3000 & isolator stiffness & {$[\mathrm{Nm} / \mathrm{rad}]$} \\
\hline$m_{\text {bus }}$ & $0.3 \mathrm{e} 3$ & $\mathrm{~S} / \mathrm{C}$ bus mass & [kg] \\
\hline$K_{z p e t}$ & $0.9 \mathrm{e} 8$ & petal hinge stiffness & {$[\mathrm{N} / \mathrm{m}]$} \\
\hline$t_{s p}$ & 0.003 & spider wall thickness & {$[\mathrm{m}]$} \\
\hline$I_{s s}$ & $0.8 \mathrm{e}-8$ & sunshield m.o.inertia & {$\left[\mathrm{m}^{4}\right]$} \\
\hline$I_{\text {propt }}$ & 5.11 & propulsion inertia & {$\left[\mathrm{kgm}^{2}\right]$} \\
\hline$\zeta$ & 0.005 & modal damping & {$[-]$} \\
\hline \multicolumn{4}{|c|}{ optics parameters } \\
\hline $\bar{\lambda}$ & $1 e-6$ & optical wavelength & {$[\mathrm{m}]$} \\
\hline$R_{o}$ & 0.98 & optical transmissivity & {$[-]$} \\
\hline$Q E$ & 0.80 & quantum efficiency & {$[-]$} \\
\hline$M_{g s}$ & 15.0 & guide star magnitude & [mag] \\
\hline \multicolumn{4}{|c|}{ controls parameters } \\
\hline$\overline{f_{c a}}$ & 0.01 & ACS control bandwidth & {$[\mathrm{Hz}]$} \\
\hline$K_{c}$ & 0.0 & FSM/ACS coupling & {$[0-1]$} \\
\hline$K_{c f}$ & 2000 & FSM controller gain & {$[-]$} \\
\hline
\end{tabular}

\section{B. Disturbance Analysis}

A disturbance analysis was carried out with the initial parameters, $p_{o}$, given in Table I. Results for LOS jitter are contained in Figure 7 . The bottom plot shows a sample time realization for 5 seconds and the centroid $\mathrm{X}$ location. The middle plot shows the power spectral density (PSD) of LOS jitter (RSS LOS) for a frequency domain and time domain calculation. The top plot is the cumulative RSS of LOS jitter as a function of frequency.
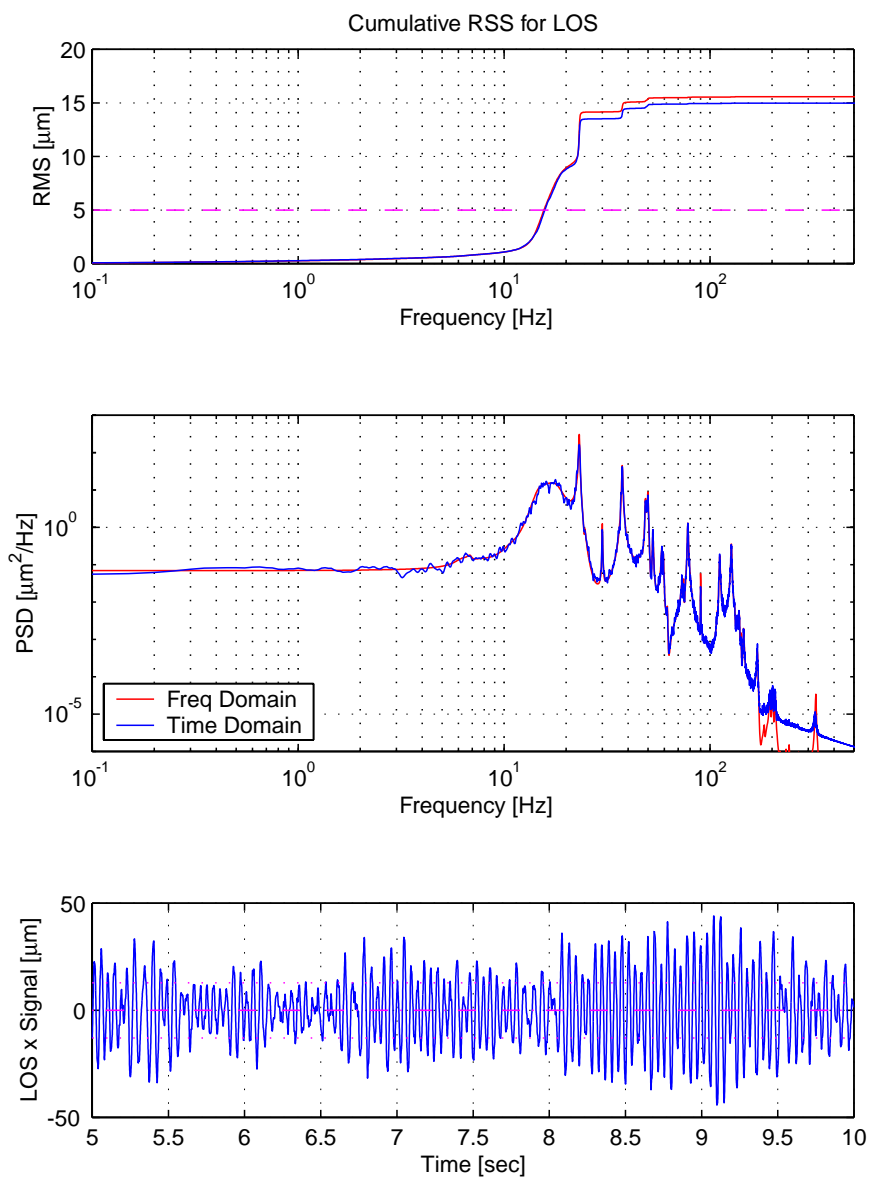

Fig. 7. LOS Jitter initial disturbance analysis

Another way to look at performance $J_{z, 2}$ is to plot the time histories from the motions of centroid $\mathrm{X}$ and $\mathrm{Y}$ versus each other. This has been done in Figure 8. The predicted RSS LOS is $14.97 \mu \mathrm{m}$, versus a requirement of $5 \mu \mathrm{m}^{7}$.

The wavefront error performance is omitted here for simplicity, but it is discussed in de Weck's Ph.D. thesis [14]. Table II shows an overview of the predicted performance, using the initial parameters $p_{o}$.

The wavefront error requirement $(\lambda / 50)$ is nearly met, but the pointing performance has to improve by a factor of roughly three.

${ }^{7}$ This requirement comes from the assumption of $25 \mu \mathrm{m}$ pixel pitch and a desire to maintain RSS LOS jitter below $1 / 5$ of a pixel. 


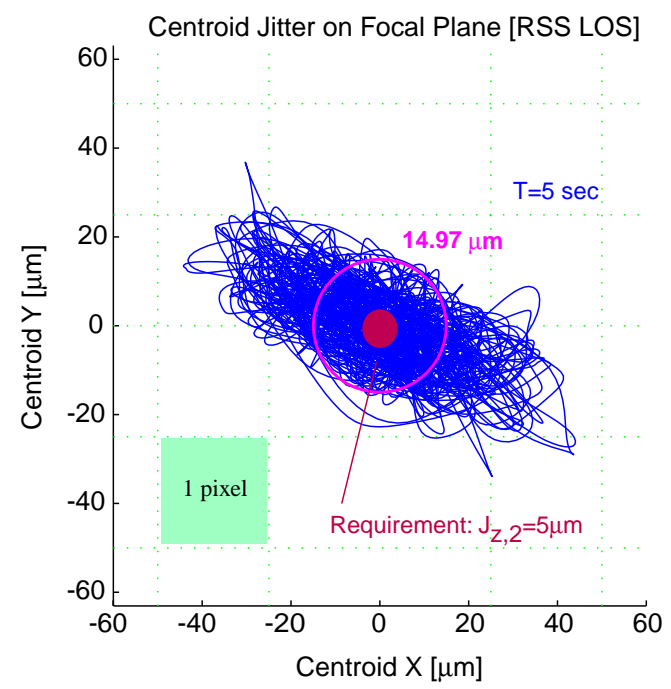

Fig. 8. RSS LOS Centroid Jitter Plot on Focal Plane

TABLE II

Initial Performance Analysis Results

\begin{tabular}{lllll}
\hline \hline Performance & Lyap & Time & Req & Units \\
\hline$J_{z, 1}$ RMMS WFE & 25.61 & 19.51 & 20 & {$[\mathrm{~nm}]$} \\
$J_{z, 2}$ RSS LOS & 15.51 & 14.97 & 5 & {$[\mu \mathrm{m}]$} \\
\hline \hline
\end{tabular}

\section{Sensitivity Analysis}

The next step is a sensitivity analysis for the 25 variable design parameters of NEXUS. The sensitivity produces the normalized Jacobian matrix evaluated at the initial design point $p_{o}$,

$$
\bar{\nabla} J_{z}=\frac{p_{o}}{J_{z, o}}\left[\begin{array}{cc}
\frac{\partial J_{z, 1}}{\partial R_{u}} & \frac{\partial J_{z, 2}}{\partial R_{u}} \\
\cdots & \cdots \\
\frac{\partial J_{z, 1}}{\partial K_{c f}} & \frac{\partial J_{z, 2}}{\partial K_{c f}}
\end{array}\right]
$$

which is graphically shown in Figure 9 . Note that parameters $R_{u}$ through $T_{g s}$ are disturbance parameters, $m_{S M}$ through $\zeta$ are structural plant parameters, $\lambda$ through $M_{g s}$ are optical parameters and $f_{c a}$ through $K_{c f}$ are control parameters.

The RMMS WFE is most sensitive to the upper operational wheel speed, $R_{u}$, the RWA isolator stiffness, $K_{r I S O}$, and the deployable petal hinge stiffness, $K_{z p e} t$. The RSS LOS is most sensitive to the dynamic wheel imbalance, $U_{d}$, the RWA isolator stiffness, $K_{r I S O}$, structural damping, $\zeta$, the guide star magnitude, $M_{g s}$ and the FSM (fine pointing loop) control gain, $K_{c f}$. Interpreting these results one would expect for example that a $1.0 \%$ decrease in the isolator stiffness, $K_{r I S O}$ should lead to roughly a $1.5 \%$ decrease in LOS jitter. The sensitivity analysis can be used to select a subset of interesting parameters for further analysis.
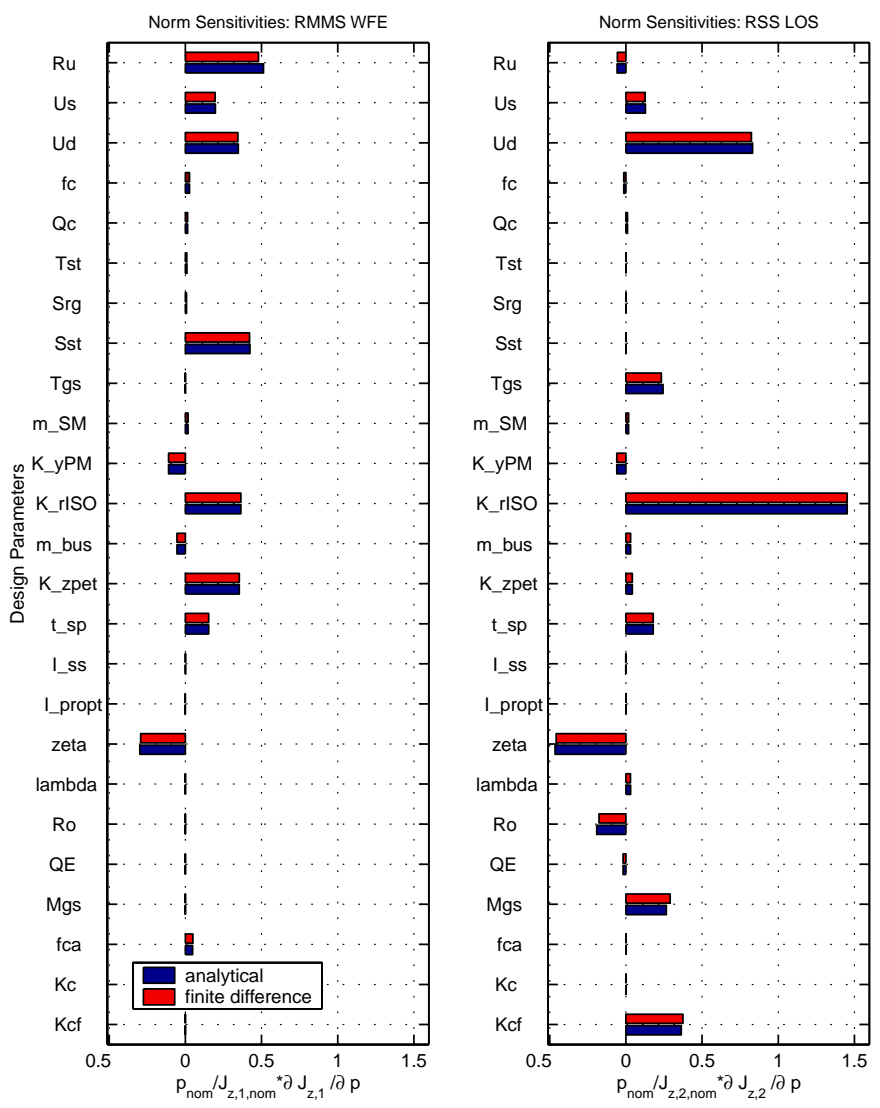

Fig. 9. NEXUS normalized sensitivity analysis results at $p^{o}$.

\section{Bivariate Isoperformance}

A bivariate isoperformance analysis is conducted for NEXUS using $J_{z, 1}=$ RSS LOS as the performance and the two most sensitive parameters from Figure 9 (right column) as the parameters. Hence, dynamic wheel imbalance, $U_{d}$, is traded versus RWA isolator joint stiffness, $K_{r I S O}$, while constraining the performance to the requirement level, $J_{z, 2, r e q}=5[\mu \mathrm{m}]$. The results are contained in Figure 10

The isoperformance contour at RSS LOS $=5 \mu \mathrm{m}$ can be reached from the initial design, $p_{o}$, by keeping the same amount of imbalance in the wheels (specification value of reaction wheel: $\left.U_{d}=60\left[\mathrm{gcm}^{2}\right]\right)$ and softening the isolator to below 1000 [Nm/rad], thus reducing the isolator corner frequency to roughly $1.2 \mathrm{~Hz}$. Alternatively the isolator can remain the same and the imbalance could be reduced to close to its lower bound, $U_{d}=1\left[\mathrm{gcm}^{2}\right]$. The isoperformance contour defines a set, $\mathbf{I}$, of performance invariant solutions, $p_{\text {iso }} \in \mathbf{I}$, and passes through these two points. A combination of the two proposed changes, improved balancing and isolator softening, is likely to result in the desired effect. Note that the performance degrades significantly for stiffer isolator struts and larger imbalances. The region in the upper right of Figure 10, where LOS jitter of 160 $\mu m$ is predicted, occurs, when the isolator modes coincide with other flexible modes of the NEXUS structure. 


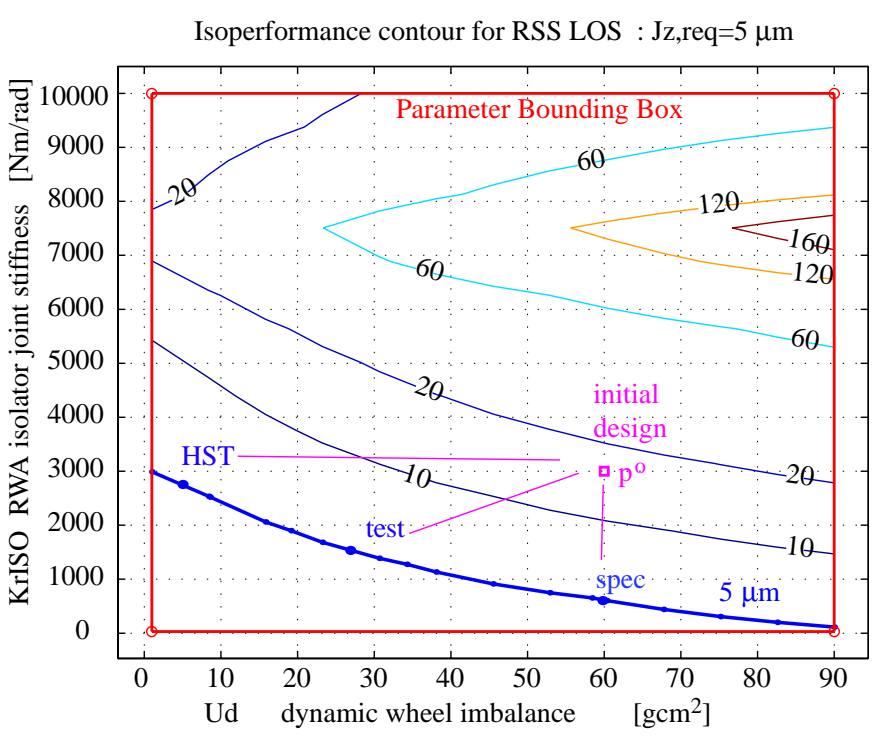

Fig. 10. NEXUS Bivariate Isoperformance analysis with $p_{1}=U_{d}$, $p_{2}=K_{r} I S O$ and $J_{z}=R S S L O S S$

\section{E. Multiobjective Optimization}

Since solutions, $p_{i s o}$, in the isoperformance set $\mathbf{I}$ do not distinguish themselves via their performance, we may satisfy some additional objectives. For the bivariate analysis in IV-D, for example, it is not immediately clear whether it is more favorable or "expensive" to improve the balancing of the reaction wheels or to build a "softer" hexapod isolator. Once the (iso)performance requirements, $J_{z}\left(p_{i s o}\right)=J_{z, r e q}$, are met one may consider competing cost objectives $J_{c}$ (control effort, implementation cost, system mass, dissipated power, etc.) or risk objectives $J_{r}$ (stability margins, sensitivity of performance to parametric uncertainty etc.). Which combination of $J_{c}$ and $J_{r}$ to use is application dependent. The result is a family of pareto optimal solutions, $p_{i s o}^{*}$, which is presented to the designer.

Such a multivariable analysis was conducted for a subset of 10 out of the 25 design parameters for NEXUS. The two performance objectives RMMS WFE and RSS LOS were defined above. The cost and risk objectives are defined as follows:

- $J_{c, 1}=$ Cost - closeness to "mid-range": $\left(p_{U B}-p_{L B}\right) / 2$

- $J_{c, 2}=$ FSM control gain

- $J_{r, 1}=$ Percent performance uncertainty

The three pareto optimal solutions, which each individually optimize one of the above objectives, while meeting the isoperformance condition, are shown in the radar ( $\mathrm{spi}-$ der) plot of Figure 11.

Specifically, the isoperformance condition leads to the fact that all designs, $p_{i s o}^{*}$, asymptote to the same value in the cumulative RMS plot, as shown for RSS LOS in

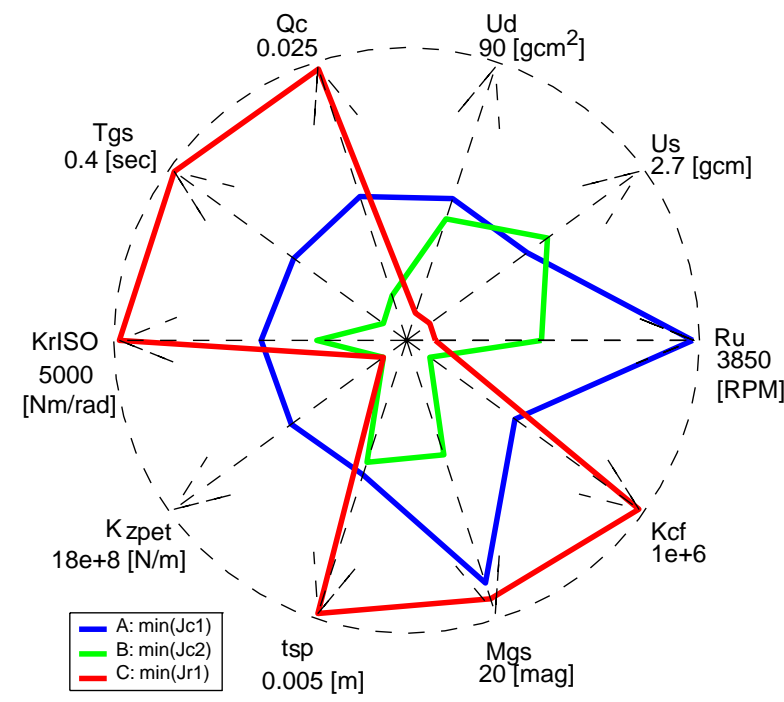

Fig. 11. NEXUS Multivariable Isoperformance. Radar plot of 3 pareto optimal designs.

Reference [14]. The results for the NEXUS pareto optimal designs are summarized in Table III.

TABLE III

NEXUS PARETO OPTIMAL DESIGNS

\begin{tabular}{l|ll|lll}
\hline \hline & $J_{z, 1}$ & $J_{z, 2}$ & $J_{c, 1}$ & $J_{c, 2}$ & $J_{r, 1}$ \\
\hline $\mathrm{A}$ & 20.0000 & 5.2013 & 0.6324 & 0.4668 & $\pm 14.3 \%$ \\
$\mathrm{~B}$ & 20.0012 & 5.0253 & 0.8960 & 0.0017 & $\pm 8.8 \%$ \\
$\mathrm{C}$ & 20.0001 & 4.8559 & 1.5627 & 1.0000 & $\pm 5.3 \%$ \\
\hline \hline
\end{tabular}

Even though these designs achieve the same WFE and LOS jitter performance, their dominant contributors in terms of disturbance sources are likely different. This leads naturally to the application of isoperformance for dynamics error budgeting.

\section{F. Error Budgeting}

Error budgeting finds a design, which balances the error contributions from all expected sources (e.g. reaction wheel imbalances, sensor noise) and assesses the feasibility of an apriori allocation. Table IV shows the apriori allocation and the actual disturbance contributions to the variance of RSS LOS for Design "A", which is chosen as the final design, $p_{i s o}^{* *}$.

The error budget can be expressed in terms of the fractional contribution of the $\mathrm{j}$-th disturbance source to the i-th performance as

$$
\Psi_{i}=\sum_{j=1}^{n_{d}} \Psi_{i, j}=J_{z, r e q, i}^{2}
$$

The relative contributions to the performance can be shown by plotting the fractional contributions of the $\mathrm{j}$-th 
TABLE IV

NEXUS ERROR BUDGET

\begin{tabular}{lllll}
\hline \hline Error Source & VAR \% & Budget & VAR \% & Capability \\
\hline RWA & 50.00 & 3.54 & 0.92 & 0.499 \\
Cryocooler & 25.00 & 2.50 & 0.22 & 0.244 \\
ACS Noise & 5.00 & 1.12 & 0.00 & $7 \mathrm{E}-6$ \\
GS Noise & 20.00 & 2.24 & 98.8 & 5.172 \\
\hline Total & 100 & 5.00 & 100 & 5.2013 \\
\hline \hline
\end{tabular}

error source on a sphere (not showing ACS noise). This sphere is called the Error Sphere, see Figure 12. One sees that for design "A" the dominant noise source is guide star noise. This is desirable, since now the pointing performance is limited by the faintness of guide stars and not by mechanical imperfections of the hardware.

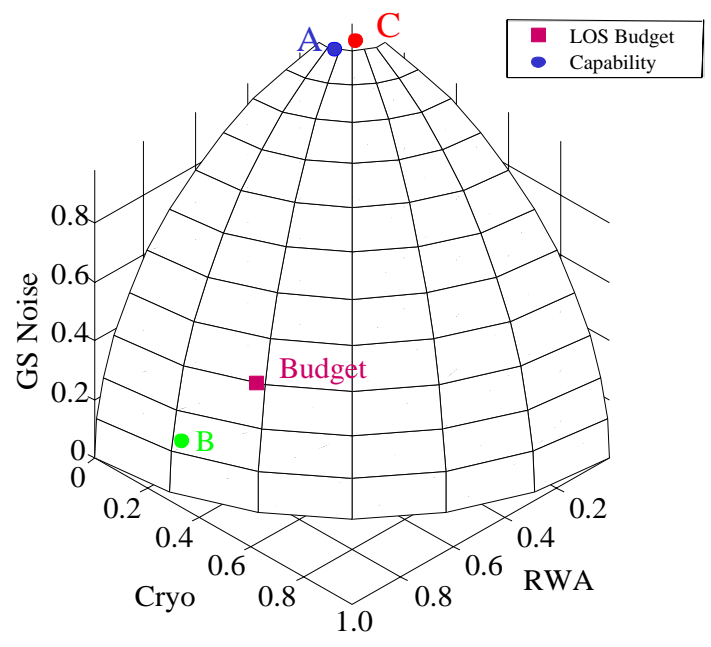

Fig. 12. NEXUS Error Sphere for RSS LOS. Note: ACS sensor noise contributions not shown.

Error Budgeting is an obvious application of isoperformance, since an apriori error budget will always result in the desired performance level. The advantage of using isoperformance in this context is that a "capability" error budget, $\Psi^{* *}$, can be found, which is theoretically achievable since it is based on the underlying integrated model.

Figure 13 shows the parameters values for the final design "A". The improvements, compared to the initial design $p_{o}$ are achieved by a well balanced mix of changes in the disturbance parameters, structural redesign and increase in control gain of the FSM fine pointing loop. A comparison of the initial and "final" parameters is shown in the upper right hand corner. The optical telescope assembly (OTA) is shown on the left side and a comparison of the XY-jitter between the initial design and final design can be seen in the lower right corner. Thus, the DOCS framework was helpful in analyzing the dynamic performance of the system, in understanding critical design pa-

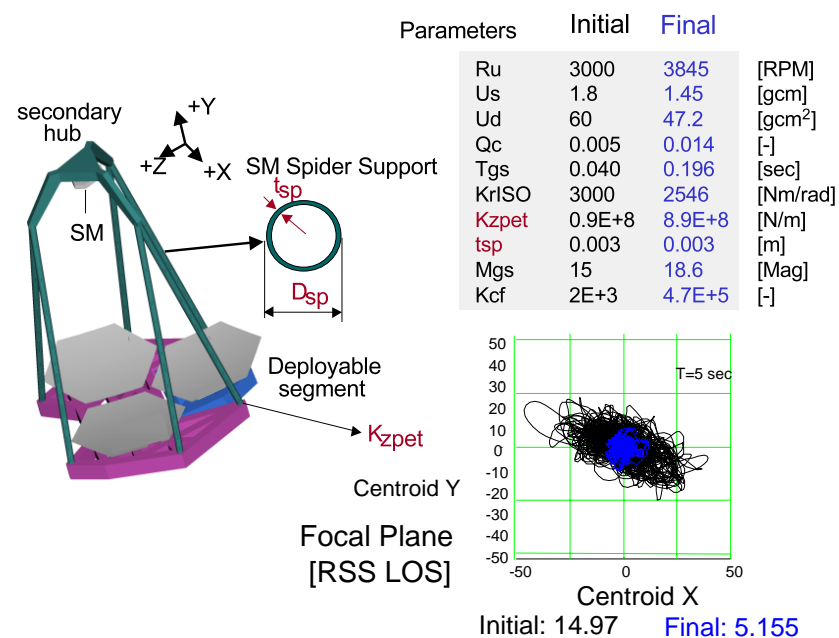

Fig. 13. NEXUS Final Design.

rameters and in finding an acceptable design, where the "burden" has been evenly distributed in the system.

\section{COnClusions}

DOCS is a comprehensive framework for modeling, simulation and analysis of space telescopes. While certainly not representing the only approach to integrated modeling of the dynamic aspects of telescopes, it has proven versatile and efficient for a number of missions such as SIM, NGST, TPF and NEXUS. The key steps in integrated modeling and simulation are subsystem modeling, model assembly and conditioning, performance assessment (=disturbance analysis), uncertainty analysis, sensitivity analysis, redesign, design optimization and isoperformance. A comprehensive NEXUS spacecraft analysis was conducted to demonstrated the types of results and insights that can be obtained. It was demonstrated that the tight pointing and phasing requirements for the telescope can be achieved by a well "balanced" design that distributes the burden between the participating subsystems. Despite the progress made in past years, significant challenges remain:

1. Enable fast simulations for large models

2. Analytical sensitivities for all parameters

3. Incorporation of thermal or impulsive transients

4. Effective use of experimental results

\section{ACKNOWLEDGMENTS}

This research was supported by the NASA Goddard Space Flight Center under contracts No. NAG5-6079 and No. NAG5-7839 and by the Jet Propulsion Laboratory under the SIM research contract No. JPL 961123. The above research contracts were monitored by Mr. Gary Mosier (GSFC), Dr. Sanjay Joshi (JPL) and Mr. Robert Grogan (JPL), respectively. Financial support to the first co-author was further provided in the form of a Pellegrini Medicus fund scholarship and a Carroll L. Wilson award. 


\section{Appendix A: Mathematical Background}

The kernel of the current disturbance, sensitivity and uncertainty analysis framework was originally developed by Gutierrez [25]. It is a powerful methodology for predicting the root-mean-square (RMS) ${ }^{8}$ of the performance outputs, $z$, recast as the performance metrics $J_{z}$. This performance prediction can occur using the time domain, PSD or Lyapunov approach. Additionally a Lagrange multiplier approach is used to obtain the (exact) analytical sensitivities. The computation of sensitivities in the timedomain or frequency domain has not yet been explored and remains for future work. The uncertainty analysis approach used in this paper is the first order approach. The purpose of this appendix is to provide a short summary of the framework and the mathematics presented in [25], thus allowing this paper to be self-contained and clearly establishing a theoretical foundation.

\section{A. Disturbance Modeling}

A disturbance analysis is required, when deterministic or stochastic disturbances $w(t)$ are present and it is unclear whether the performances $J_{z}$ will remain within a required value $J_{z, \text { req }}$. Note that generally $w$ and $J_{z}$ will be vectors. The disturbances $w(t)$ can be undesired forces, torques, base motion, sensor and actuator noise, among others. The correlation function of the random process $w(t)$ is defined as

$$
R_{w w}\left(t_{1}, t_{2}\right)=E\left[w\left(t_{1}\right) w^{T}\left(t_{2}\right)\right]
$$

where $E[]$ is the expectation operator and $w(t)$ is a vector of stochastic random processes. If $w(t)$ is stationary the values of $w(t)$ will change over time, but the statistics of $w(t)$ will not and $R_{w w}$ is a function of the single time-lag $\tau$.

$$
R_{w w}(\tau)=E\left[w(t) w^{T}(t+\tau)\right]
$$

Assuming that all $w(t)$ are zero-mean, the covariance matrix $\Sigma_{w}$ of the disturbance signals is the value of the correlation matrix $R_{w w}$ for $\tau=0$.

$$
\begin{aligned}
& \Sigma_{w}=R_{w w}(0)=\left[\begin{array}{cccc}
E\left[w_{1}^{2}\right] & E\left[w_{1} w_{2}\right] & \cdots & E\left[w_{1} w_{n}\right] \\
E\left[w_{2} w_{1}\right] & E\left[w_{2}^{2}\right] & \cdots & E\left[w_{2} w_{n}\right] \\
\vdots & \vdots & \ddots & \vdots \\
E\left[w_{n} w_{1}\right] & E\left[w_{n} w_{2}\right] & \cdots & E\left[w_{n}^{2}\right]
\end{array}\right. \\
& =\left[\begin{array}{cccc}
\sigma_{w_{1}}^{2} & \sigma_{w_{2} w_{1}} & \cdots & \sigma_{w_{1} w_{n}} \\
\sigma_{w_{2} w_{1}} & \sigma_{w_{2}}^{2} & \cdots & \sigma_{w_{2} w_{n}} \\
\vdots & \vdots & \ddots & \vdots \\
\sigma_{w_{n} w_{1}} & \sigma_{w_{n} w_{1}} & \cdots & \sigma_{w_{n}}^{2}
\end{array}\right]
\end{aligned}
$$

\footnotetext{
${ }^{8}$ or root-sum-square (RSS), root-mean-mean-square (RMMS)
}

The simplification in the covariance matrix $\Sigma_{w}$ can be made for zero-mean processes, since for a typical term $\sigma_{w_{i} w_{j}}$ in the covariance matrix we can write

$$
\begin{aligned}
& \sigma_{w_{i} w_{j}}=E\left[\left(w_{i}-\mu_{w_{i}}\right)\left(w_{j}-\mu_{w_{j}}\right)\right]= \\
& E\left[w_{i} w_{j}\right]-\underbrace{E\left[w_{i} \mu_{w_{j}}\right]}_{=0}-\underbrace{E\left[\mu_{w_{i}} w_{j}\right]}_{=0}+\underbrace{E\left[\mu_{w_{i}} \mu_{w_{j}}\right]}_{=0}=
\end{aligned}
$$$$
E\left[w_{i} w_{j}\right]=\sigma_{w_{i} w_{j}}
$$

where $\mu_{w_{i}}=E\left[w_{i}\right]$ is the mean (expected value) of the $\mathrm{i}$-th random process. The mean-square values of the elements of $w$ are simply the diagonal entries in the covariance matrix.

$$
\left(\Sigma_{w}\right)_{i, i}=E\left[w_{i}^{2}(t)\right]
$$

where $w_{i}(t)$ is the $\mathrm{i}$-th element in $w$. If $w$ is zero-mean, then the mean-square values and the variances are identical.

$$
\sigma_{w_{i}}^{2}=E\left[w_{i}^{2}(t)\right]-\underbrace{\left(E\left[w_{i}\right]\right)^{2}}_{=0}=\mathrm{RMS}^{2}
$$

The (power) spectral density function $S_{w w}(\omega)$ can be obtained by taking the Fourier transform of equation (4)

$$
S_{w w}(\omega)=\mathcal{F}\left[R_{w w}(\tau)\right]=\int_{-\infty}^{+\infty} R_{w w}(\tau) e^{-j \omega \tau} d \tau
$$

Note that the $1 / 2 \pi$ factor is not included in the definition of the Fourier transform. Other authors [71] place it in the Fourier transform formula. Either definition will produce the same result in the end as long as the definition is used consistently. The inverse Fourier transform of $S_{w w}(\omega)$ recovers the correlation function.

$$
R_{w w}(\tau)=\mathcal{F}^{-1}\left[S_{w w}(\omega)\right]=\frac{1}{2 \pi} \int_{-\infty}^{+\infty} S_{w w}(\omega) e^{+j \omega \tau} d \omega
$$

Evaluating (10) at $\tau=0$ will produce the covariance matrix of $w$.

$$
R_{w w}(0)=\Sigma_{w}=\frac{1}{2 \pi} \int_{-\infty}^{+\infty} S_{w w}(\omega) d \omega
$$

Equation (11) suggests an alternative way of calculating the mean-square values of $w$ by integrating under the spectral density functions, namely

$$
\sigma_{w_{i}}^{2}=\left[\Sigma_{w}\right]_{i, i}=\frac{1}{2 \pi} \int_{-\infty}^{+\infty}\left[S_{w w}(\omega)\right]_{i, i} d \omega
$$

The diagonal elements of the spectral density function matrix $S_{w w}(\omega)$ are usually referred to as power spectral densities (PSD's), whereas the off-diagonal elements are the cross spectral densities. Equation (12) states that the variance is equal to the area beneath the PSD scaled by a 
factor of $\frac{1}{2 \pi}$. Depending on the shape of the PSD's representing $w$ it is possible to approximate the shape of the functions $S_{w w}(\omega)$ by pre-whitening filters in state space form, where the inputs to the state space system are unit intensity white noise processes $d$ :

$$
\begin{aligned}
& \dot{q}_{d}=A_{w d} q_{d}+B_{w d} d \\
& w=C_{w d} q_{d}+D_{w d} d
\end{aligned}
$$

Note that the feedthrough matrix $D_{w d}$ is generally zero, since white noise will otherwise feed through, which is not physically realizable since an ideal white noise process has infinite energy.

\section{B. Integrated Modeling}

The plant, optics and control loops are modeled in a continuous linear time-invariant model as:

$$
\begin{aligned}
& \dot{q}_{p}=A_{p} q_{p}+B_{z w} w+B_{z r} r \\
& z=C_{p} q_{p}+D_{z w} w+D_{z r} r
\end{aligned}
$$

where $q_{p}$ is the appended state vector, $r$ is the vector of reference inputs (commands) and $z$ is a vector of performance outputs, from which the actual performance metrics $J_{z}$ are formed. If the disturbances are cast in state space form as suggested in (13), they can be appended into (14). This overall state space system (15) then becomes the "integrated model" of the opto-mechanical system representing the closed or open loop dynamics and is shown in Figure 14. In steady-state the reference commands are

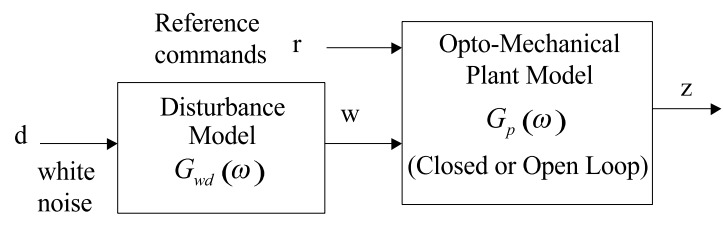

Fig. 14. General block diagram of system dynamics from white noise disturbances $d$ to system performance signals $z$.

often $r=0$ and generally the feedthrough matrix $D_{z d}$ is also zero, since otherwise the white-noise input $d$ will lead to an infinite variance $\sigma_{z_{i}}^{2}$ of the i-th performance signal $z_{i}$ that is affected by the feedthrough.

$$
\begin{aligned}
& \dot{q}=A_{z d} q+B_{z d} d+B_{z r} r \\
& z=C_{z d} q+D_{z d} d+D_{z r} r
\end{aligned}
$$

or in matrix S-form

$$
S_{z d}=\left[\begin{array}{cc}
A_{z d} & B_{z d} \\
C_{z d} & D_{z d}
\end{array}\right]
$$

In this paper it is assumed that the feedthrough matrix $D_{z d}$ is always zero. The vector $q$ represents the state vector of length $n_{s}$ and has to be ordered similar to the convention defined in [25, eq.(4.5)] as $q=\left[\begin{array}{llll}q_{d} & q_{p} & q_{c} & q_{z}\end{array}\right]^{T}$, where $q_{d}$ are the disturbance filter states, $q_{p}$ are the (structural) plant states, $q_{c}$ are the controller states and $q_{z}$ are performance weighting states, if applicable.

\section{Performance Assessment}

Once an integrated model of a nominal system design is available, the next step is to assess the performance when the model is subjected to anticipated disturbances. In this paper we will consider $\mathcal{H}_{2}$ performance metrics according to Zhou [73] as follows:

$J_{z}=\mathrm{E}\left[z^{T} z\right]^{1 / 2}=\left(\frac{1}{T} \int_{0}^{T} z(t)^{2} d t\right)^{1 / 2}$

RMS

$J_{z}=\left[\sum_{i=1}^{N} \mathrm{E}\left[z_{i}^{T} z_{i}\right]\right]^{1 / 2}=\left(\sum_{i=1}^{N} \frac{1}{T} \int_{0}^{T} z_{i}(t)^{2} d t\right)^{1 / 2}$

RSS

$J_{z}=\left[\frac{1}{N} \cdot \sum_{i=1}^{N} \mathrm{E}\left[z_{i}^{T} z_{i}\right]\right]^{1 / 2}=\left(\frac{1}{N} \cdot \sum_{i=1}^{N} \frac{1}{T} \int_{0}^{T} z_{i}(t)^{2} d t\right)^{1 / 2}$

RMMS

The RMS (root-mean-square) metric is typically used to describe the "on-average" optical pathlength difference (OPD) in an interferometer. The RSS (root-sum-squared) can be employed to describe the line-of-sight (LOS) jitter of an observatory in xy-coordinates on the focal plane and, for example, the RMMS (root-mean-mean squared) metric is used to describe the "mean" wavefront error of a light bundle by averaging the phase errors of all rays modeled by an optical ray tracing program. Three disturbance analysis approaches are discussed below.

\section{C.1 Time Domain Analysis}

A linear time-invariant system from (colored) disturbances $w$ to performances $z$ is given as:

$$
\begin{aligned}
& \dot{q}_{p}=A_{z w} q_{p}(t)+B_{z w} w(t) \\
& z(t)=C_{z w} q_{p}(t)+D_{z w} w(t)
\end{aligned}
$$

where $q_{p}$ consists of structural states and compensator states, if applicable. Equivalently, the system can be described in the frequency-domain by the transfer function matrix

$$
G_{z w}(\omega)=C_{z w}\left[j \omega I-A_{z w}\right]^{-1} B_{z w}+D_{z w}
$$


The disturbances, $w$, are the inputs to the system, while the performances, $z$, are the outputs of interest. When measured time histories of the disturbances $w(t)$ exist $^{9}$, they can be used for time integration of the state space equations (18). Once the initial condition on the state vector, $q_{p}(0)$, is specified, numerical integration of (18) can then be performed to obtain estimates of the performance time histories $z(t)$. The standard difference method technique approximates the continuous first-order equation (18) with a difference equation such as

$$
\begin{aligned}
& \frac{\left(q_{p}\right)_{n+1}-\left(q_{p}\right)_{n}}{\Delta t}=A_{z w}\left(q_{p}\right)_{n}+B_{z w} w_{n} \\
& z_{n}=C_{z w}\left(q_{p}\right)_{n}+D_{z w} w_{n}
\end{aligned}
$$

The state vector $\left(q_{p}\right)_{n+1}$ at the $n+1$-th time step can be found by the forward Euler method as

$$
\left(q_{p}\right)_{n+1}=\left[\Delta t A_{z w}+I\right]\left(q_{p}\right)_{n}+\Delta t B_{z w} w_{n}
$$

This integration method is simple but can diverge easily when $\Delta t \geq \Delta t_{\text {crit }}$. For time integrations in this paper it was found that the results obtained with the ode $45 . \mathrm{m}$ solver according to Dormand and Prince [16] gave the best results. An advantage of the time-domain disturbance analysis is that transient effects can be observed, the compliance with time-domain performance specifications can be examined and threshold crossings can be determined from the time simulation. Generally the time domain analysis is computationally more expensive than the other methods. Other disadvantages are the dependency of the answers on the initial conditions of the state vector $q_{p}(0)$ and on the seed used for the white noise random number generators.

\section{C.2 Frequency Domain Analysis}

For linear systems in the time domain, the output can be expressed as a convolution of the input with the impulseresponse function of the system. In the frequency domain (i.e. Laplace domain), the output is equal to the input multiplied by the transfer function (matrix). The disturbance spectral density matrix $S_{w w}(\omega)$ can be measured experimentally or obtained from a shaping (prewhitening) filter as $S_{w w}(\omega)=G_{d}(\omega) G_{d}^{H}(\omega)$. The performance spectral density matrix $S_{z z}$ can be obtained from [71]

$$
S_{z z}(\omega)=G_{z w}(\omega) S_{w w}(\omega) G_{z w}^{H}(\omega)
$$

where $S_{w w}$ is the disturbance spectral density matrix discussed above and $G_{z w}$ is the open or closed loop plant transfer function matrix from (19). $S_{w w}(\omega)$ can be a continuous function of frequency, or for the case of disturbances consisting of a series of discrete harmonics, it

\footnotetext{
${ }^{9}$ These can be obtained from spinup tests of reaction wheels, vibration testing of cryocoolers or noise floor measurements on sensors, among others.
}

can contain impulses at the frequencies of the harmonics. In the latter case, $S_{z z}(\omega)$ will also contain a series of impulses. $S_{z z}(\omega)$ provides information on the frequency content of the performances. The covariance matrix of the performances $\Sigma_{z}$ (for zero-mean processes) is obtained as

$$
\Sigma_{z}=\frac{1}{2 \pi} \int_{-\infty}^{+\infty} S_{z z}(\omega) d \omega=\int_{-\infty}^{+\infty} S_{z z}(f) d f
$$

The variance of the i-th performance is therefore given by

$$
\begin{aligned}
& \sigma_{z_{i}}^{2}=\left[\Sigma_{z}\right]_{i, i}=\frac{1}{2 \pi} \int_{-\infty}^{+\infty}\left[S_{z z}(\omega)\right]_{i, i} d \omega= \\
& \int_{-\infty}^{+\infty}\left[S_{z z}(f)\right]_{i, i} d f=2 \int_{0}^{+\infty}\left[S_{z z}(f)\right]_{i, i} d f
\end{aligned}
$$

Taking the square root of $\sigma_{z_{i}}^{2}$ produces the root-meansquare (RMS) value. It is important to specify whether a PSD is one or two sided and given in $\mathrm{Hz}$ or rad/sec [71]. In practice the upper and lower frequency integration limits are $f_{\min }$ and $f_{\max }$, respectively.

$$
\sigma_{z_{i}}^{2} \approx 2 \int_{f_{\min }}^{f_{\max }}\left[S_{z z}(f)\right]_{i, i} d f
$$

It is important to ensure that the frequency range that contributes most to the RMS value is sufficiently captured within these limits. One way to verify this is by computing the cumulative RMS function $\sigma_{z_{i}, c}\left(f_{o}\right)$ as

$$
\sigma_{z_{i}, c}\left(f_{o}\right)=\left[2 \int_{f_{\min }}^{f_{o}}\left[S_{z z}(f)\right]_{i, i} d f\right]^{\frac{1}{2}}
$$

where $f_{o} \in\left[f_{\min } \ldots f_{\max }\right]$. If most of the energy lies in this range, then $\sigma_{z_{i}, c}\left(f_{\max }\right)$ should be very close to the true value of $\sigma_{z_{i}}$. Generally, the frequency-domain approach is more efficient than a time-domain analysis. The method however requires high frequency resolution near lightly damped modes in order to arrive at correct RMS values. Also the frequency domain method is not well suited to assess the transient performance of a linear timeinvariant system.

\section{C.3 Lyapunov Analysis}

The third type of disturbance analysis can be conducted if the disturbances $w$ are modeled as the outputs of a shaping filter in the form of equation (13). In order to keep the disturbance $w$ from having infinite energy, there should be no feedthrough matrix $D_{w d}$. The state space system (13) containing the disturbance dynamics is appended to the plant model (14) resulting in the overall system model (15). If the system is asymptotically stable, the state covariance matrix obeys the Lyapunov equation [21].

$$
A_{z d} \Sigma_{q}+\Sigma_{q} A_{z d}^{T}+B_{z d} B_{z d}^{T}=\dot{\Sigma}_{q}
$$

In order to do time integration of the above dynamics, the initial state covariance, $\Sigma_{q_{o}}$, would have to be specified. Since the white noise disturbance processes $d$ are 
assumed to be stationary and the reference commands $r$ have been set to zero, the statistics of the state vector are also stationary and $\dot{\Sigma}_{q}=0$. This is not true for transient processes. One may then solve the steady-state Lyapunov equation of order $n_{s}$ for the state covariance matrix $\Sigma_{q}$ of the system (15).

$$
A_{z d} \Sigma_{q}+\Sigma_{q} A_{z d}^{T}+B_{z d} B_{z d}^{T}=0
$$

For $i=1,2, \ldots, n_{z}$ one solves for each RMS value by extracting the i-th row from the $C_{z d}$ matrix, pre- and postmultiplying $\Sigma_{q}$ and by taking the square root. The RMS of the $\mathrm{i}$-th performance metric is then given as:

$$
\sigma_{z_{i}}=\left(C_{z d, i} \Sigma_{q} C_{z d, i}^{T}\right)^{1 / 2}
$$

where $C_{z d, i}$ is the vector formed by the $\mathrm{i}$-th row of the $C_{z d}$ matrix and $\Sigma_{q}$ is the state covariance matrix of the state space system that obeys the steady-state Lyapunov equation in (28). Alternatively one can pre- and post-multiply with the entire $C_{z d}$ matrix to obtain the performance covariance matrix $\Sigma_{z}$.

$$
\begin{aligned}
& \Sigma_{z}=E\left[z z^{T}\right]=E\left[C_{z d} q q^{T} C_{z d}^{T}\right]=C_{z d} E\left[q q^{T}\right] C_{z d}^{T} \\
& =C_{z d} \Sigma_{q} C_{z d}^{T}
\end{aligned}
$$

The variances of the individual performances (RMS squared) are then contained on the diagonal of $\Sigma_{z}$, where $\Sigma_{z}$ is of the form

$$
\Sigma_{z}=\left[\begin{array}{cccc}
\sigma_{z_{1}}^{2} & \sigma_{z_{1} z_{2}} & \cdots & \sigma_{z_{1} z_{n}} \\
\sigma_{z_{2} z_{1}} & \sigma_{z_{2}}^{2} & \cdots & \sigma_{z_{2} z_{n}} \\
\vdots & \vdots & \ddots & \vdots \\
\sigma_{z_{n} z_{1}} & \sigma_{z_{n} z_{2}} & \cdots & \sigma_{z_{n}}^{2}
\end{array}\right]
$$

and $n$ is the total number of elements in the performance vector $z$. Thus, the Lyapunov method provides a relatively direct way of arriving at the RMS estimates (in the sense of statistical steady state) by solving one matrix equation (28) and computing a matrix triple product (30).

One problem is that the computational cost of solving (28) increases as $\sim n_{s}^{3}$, where $n_{s}$ is the number of states. If the performance metric of interest is a weighted sum of the performance outputs $z$ and the $z_{i}$ in (31) represent these weighted outputs, then the scalar performance cost $J_{z}$ can be defined as

$$
J_{z}=\operatorname{trace}\left[\Sigma_{z}\right]=\sigma_{z_{1}}^{2}+\sigma_{z_{2}}^{2}+\cdots+\sigma_{z_{n}}^{2}
$$

The percent contribution of the i-th weighted output to the overall performance cost $J_{z}$ is then

$$
\% \text { contribution }=\frac{\sigma_{z_{i}}^{2}}{J_{z}} \times 100 \%
$$

The main drawback of the Lyapunov approach is that it does not provide insight into the frequency content of the outputs. The main advantage is that the answers provided are immune to the frequency resolution and time step, $\Delta t$, problems associated with the frequency-domain and time-domain approaches, respectively. Also, analytical sensitivities can be calculated as presented in the next subsection.

\section{Sensitivity Analysis}

Determining the sensitivity of certain outputs (e.g. performance metrics) of a system with respect to model parameters can provide useful information. This information can be used for model-to-test-data correlation, performance enhancement, uncertainty analysis and isoperformance analysis. Gutierrez [25] proposes a Lagrangian approach for obtaining the sensitivities $\partial \sigma_{z} / \partial p$ or $\partial J_{z} / \partial p$ based on earlier work by Jacques [33]. Note that $p$ can be a vector of modal or physical parameters of the system. The first step, for each performance metric $z_{i}, i=1,2, \ldots, n_{z}$, is to solve for the corresponding Lagrange multiplier matrix $L_{i}$. Again a steady state Lyapunov equation of order $n_{s}$ has to be solved.

$$
L_{i} A_{z d}+A_{z d}^{T} L_{i}+C_{z d, i}^{T} C_{z d, i}=0
$$

Next the governing sensitivity equation (GSE) is solved by substituting the results from Equations (28) and (34). Additionally the matrix derivatives with respect to the parameters of interest $p_{j}, j=1,2, \ldots, n_{p}$, need to be computed. The result is the partial derivative of the variance of the i-th performance $z_{i}$ with respect to the j-th parameter $p_{j}$

$$
\begin{aligned}
& \frac{\partial \sigma_{z_{i}}^{2}}{\partial p_{j}}=\operatorname{trace}\left[\Sigma_{q} \frac{\partial\left(C_{z d, i}^{T} C_{z d, i}\right)}{\partial p_{j}}\right]+ \\
& \operatorname{trace}\left[L_{i}\left\{\frac{\partial A_{z d}}{\partial p_{j}} \Sigma_{q}+\Sigma_{q} \frac{\partial A_{z d}^{T}}{\partial p_{j}}+\frac{\partial\left(B_{z d} B_{z d}^{T}\right)}{\partial p_{j}}\right\}\right]
\end{aligned}
$$

Here $L_{i}$ is the Lagrange multiplier matrix for the i-th performance. The Lagrange multiplier matrix obeys the dual Lyapunov equation given in (34). Consult References [25] and [33] for a detailed derivation of the GSE. The above equation gives the sensitivity of the variance $\sigma_{z_{i}}^{2}$, but usually the sensitivity with respect to the RMS is desired. The results from (29) and (35) are substituted in

$$
\frac{\partial \sigma_{z_{i}}}{\partial p_{j}}=\frac{1}{2 \sigma_{z_{i}}} \cdot \frac{\partial \sigma_{z_{i}}^{2}}{\partial p_{j}}
$$

in order to obtain the desired sensitivity. Normalization with a factor $p_{j, n o m} / \sigma_{z_{i}, \text { nom }}$ is optional. This normalization allows comparing sensitivities with respect to param- 
eters of different units:

$\frac{p_{n o m}}{\left(\sigma_{z_{i}}\right)_{\text {nom }}} \frac{\partial \sigma_{z_{i}}}{\partial p}=\frac{\frac{\partial \sigma_{z_{i}}}{\left(\sigma_{z_{i}}\right)_{n o m}}}{\frac{\partial p}{p_{n o m}}} \approx \frac{\frac{\Delta \sigma_{z_{i}}}{\left(\sigma_{z_{i}}\right)_{n o m}}}{\frac{\Delta p}{p_{n o m}}} \approx \frac{\% \text { change in } \sigma_{\mathrm{z}_{\mathrm{i}}}}{\% \text { change in } p}$

The matrix partial derivatives such as $\partial A_{z d} / \partial p_{j}$ in (35) represent the main difficulty in finding the sensitivities for large order systems. Initial work on sensitivities for modal parameters (frequencies, damping ratio, modal mass) and physical parameter (masses, stiffnesses) is presented by Gutierrez [25].

\section{E. Isoperformance Analysis}

Isoperformance is discussed in detail by de Weck in Reference [14]. For the multivariable case three algorithms for obtaining an approximation of the isoperformance set, $\mathbf{I}$, are proposed. These are full factorial search with branchand-bound, tangential (contour) front following and vector spline approximation.

The tangential front following algorithm is a generalization of a gradient-based contour following algorithm, which is used when $n_{p}-n_{z}=1$. The idea is to gradually explore the isoperformance set $\mathbf{I}$, starting from a random initial point, $p_{n o m}$, and subsequently stepping in tangential, orthogonal directions, $v_{j}$, where $j=n_{z}+1, \ldots, n_{p}$, which lie in the null space of the Jacobian. Thus, instead of following a single curved line, the locus of performance invariant points, $p_{i s o}$, will be an $\left(n_{p}-n_{z}\right)$-dimensional surface in $\mathbb{R}^{n_{p}}$-space.

A first order Taylor approximation of the vector performance function $J_{z}$ at a point $p^{k}=\left[\begin{array}{llll}p_{1}^{k} & p_{2}^{k} & \ldots & p_{n_{p}}^{k}\end{array}\right]^{T} \in \mathbf{B}$ can be written as:

$J_{z}\left(p^{k+1}\right)=J_{z}\left(p^{k}+\Delta p\right)=J_{z}\left(p^{k}\right)+\left.\nabla J_{z}^{T}\right|_{p^{k}} \Delta p+$ H.O.T.

The Jacobian, $\nabla J_{z}$, is the matrix of first order partial derivatives of $J_{z}$ with respect to $p$ and is written as follows:

$$
\nabla J_{z}=\left[\begin{array}{cccc}
\frac{\partial J_{z, 1}}{\partial p_{1}} & \frac{\partial J_{z, 2}}{\partial p_{1}} & \cdots & \frac{\partial J_{z, n_{z}}}{\partial p_{1}} \\
\frac{\partial J_{z, 1}}{\partial p_{2}} & \frac{\partial J_{z, 2}}{\partial p_{2}} & \cdots & \frac{\partial J_{z, n_{z}}}{\partial p_{2}} \\
\vdots & \vdots & \vdots & \vdots \\
\frac{\partial J_{z, 1}}{\partial p_{n_{p}}} & \frac{\partial J_{z, 2}}{\partial p_{n_{p}}} & \cdots & \frac{\partial J_{z, n_{z}}}{\partial p_{n_{p}}}
\end{array}\right]
$$

A singular value decomposition (SVD) of the Jacobian is a key step. It provides a set of orthogonal unit-length vectors, $v_{j}$, as the columns of matrix, $V$, thus forming the column space and null space of the Jacobian. See
Reference [67] by Strang for a good introduction to vector spaces and the SVD.

$$
U \Sigma V^{T}=\nabla J_{z}^{T}
$$

The individual matrices are structured as follows:

$$
\begin{aligned}
& U=\underbrace{\left[\begin{array}{lll}
u_{1} & \cdots & u_{n_{z}}
\end{array}\right]}_{n_{z} \times n_{z}} \\
& \Sigma=\underbrace{\left[\begin{array}{llll}
\operatorname{diag}\left(\begin{array}{ccc}
n_{z} \times n_{z} \\
\sigma_{1} & \cdots & \sigma_{n_{z}}
\end{array}\right) & 0_{n_{z} \times\left(n_{p}-n_{z}\right)}
\end{array}\right]}_{n_{z} \times n_{p}}
\end{aligned}
$$

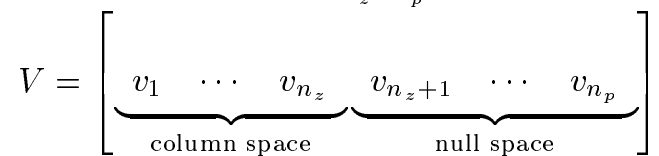

Thus, at each point there are $n_{p}-n_{z}$ directions in the null space. The corresponding vector $V_{n}$ is normal to the isoperformance surface and the vectors in $V_{t}$ are tangential to it and form the nullspace of the Jacobian. A linear combination of the vectors, $V_{t}$, in the nullspace is used to determine a tangential step, $\Delta p$, in a performance invariant direction starting from an initial point such as $p_{\text {nom }}$.

$$
\Delta p=\alpha \cdot\left(\beta_{1} v_{n_{z+1}}+\ldots+\beta_{n_{p}-n_{z}} v_{n_{p}}\right)=\alpha V_{t} \beta
$$

where $\Delta p$ is the performance invariant step increment in $\mathbb{R}^{\alpha_{1}}, \beta=\left[\beta_{1}, \ldots, \beta_{n_{p}}\right]^{T}$ is a vector of coefficients, which determines the linear combination of directions in the nullspace, $V_{t}$, and $\alpha$ is a step size.

The coefficient vector, $\beta$, is determined as follows

$$
\beta=\left\{\begin{array}{c}
\beta_{i}= \pm 1, \beta_{j}=0 \text { for } j \neq i \\
\beta_{i}= \pm \frac{1}{\sqrt{n_{p}-n_{z}}} \forall \quad i=1, \ldots, n_{p}-n_{z}
\end{array}\right.
$$

Tangential Front Following Principle generation 1

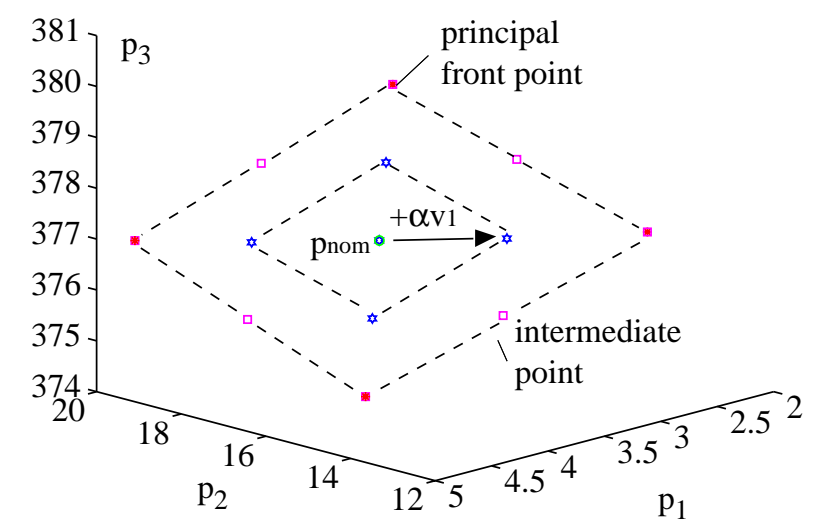

Fig. 15. Multivariable Isoperformance: Tangential Front Following principle. Axes $p_{1}, p_{2}$ and $p_{3}$ are values of some generic parameters.

The principal front points, as shown in Figure 15, propagate in one of the positive or negative directions given by 
the principal vectors, $v_{i}$, in the null space. The intermediate front points on the other hand propagate in directions which have equal contributions from all vectors in $v_{t}$. The \pm sign for each $\beta_{i}$ determines in which "quadrant" the front point propagates.

The active points form a "front", when connected to each other. The front grows gradually outwards from the initial point until the boundary is intercepted. This is analogous to "moss", which grows from an initial seed to gradually cover the entire exposed surface of an imaginary $\mathbb{R}^{n_{p}}$-dimensional rock. This is shown graphically in Figure 15. The main advantage of this algorithm, is that it converts the computational complexity from a $n_{p}$ to a $\left(n_{p}-n_{z}\right)$-dimensional problem. The underlying performance function, $J_{z}\left(p_{j}\right)$, has to be continuous and differentiable.

\section{REFERENCES}

[1] K. J. Åström And B. Wittenmark, Computer-Controlled Systems: Theory and Design, Prentice-Hall, Inc., 1997.

[2] K. J. Bathe, Finite Element Procedures, Prentice-Hall, Inc., 1996.

[3] P. R. BÉLAnger, Control Engineering, vol. 1, Saunders College Publishing, Orlando, Florida, first ed., 1995.

[4] B. BIALKE, A compilation of reaction wheel induced spacecraft disturbances, in Proceedings of the 20th Annual AAS Guidance and Control Conference, Breckenridge, CO, February 5-9, 1997. AAS Paper No. 97-038.

[5] M. Born and E. Wolf, Principles of Optics, Cambridge University Press, sixth (corrected) ed., 1998.

[6] R. G. Brown ANd P. Y. C. Hwang, Introduction to Random Signals and Applied Kalman Filtering, John Wiley \& Sons, Inc. 1997.

77] A. E. BRYSON AND R. A. Mills, Linear-quadratic-gaussian controllers with specified parameter robustness, Journal of Guidance, Control, and Dynamics, 21 (1998), pp. 11-18.

[8] M. E. CAmpbell and E. F. Crawley, Development of structural uncertainty models, Journal of Guidance, Control, and Dynamics, 20 (1997), pp. 841-849.

[9] R. D. Cook, D. S. Malkus, And M. E. Plesha, Concepts and Applications of Finite Element Analysis, John Wiley \& Sons, Inc., 1989.

[10] R. Craig JR., Structural Dynamics: An Introduction to Computer Methods, John Wiley \& Sons, Inc., 1981.

[11] S. H. Crandall and W. D. Mark, Random Vibration in Mechanical Systems, Academic Press, 1963.

[12] E. F. Crawley, B. P. Masters, and T. T. Hyde, Conceptual design methodology for high performance dynamic struc tures, in Proceedings of the $36^{\text {th }}$ AIAA Structures, Structural Dynamics, and Materials Conference, New Orleans, LA, April 1995, pp. 2768-2787. AIAA Paper No. 95-2557.

[13] L. P. DAvis, J. F. Wilson, R. E. JEwell, and J J. Roden, Hubble space telescope reaction wheel assembly vibration isolation system, in Vibration Damping Workshop, Marshall Space Flight Center, March 1986.

[14] O. L. DE WECK, Multivariable Isoperformance Methodology for Precision Opto-Mechanical Systems, PhD thesis, Massachusetts Institute of Technology, Department of Aeronautics and Astronautics, 2001.

[15] O. L. DE WeCK, D. Miller, AND H. GutierRez, Structura dynamics and controls for NGST, in Proceedings of the $34^{\text {th }}$ Liège International Astrophysics Colloquium, Liège, Belgium, June 15-18, 1998.

[16] J. R. Dormand And P. J. Prince, A family of embedded Runge-Kutta formulae, J. Comp. Appl. Math., 6 (1980), pp. 19 26.

[17] A. Dressler, HST and Beyond - Exploration and the Search for Origins: A Vision for Ultraviolet-Optical-Infrared Space
Astronomy, vol. 1, Association of Universities for Research in Astronomy, Washington D.C., 1 ed., May 1996.

[18] C. E. Eyerman And J. F. Shea, A systems engineering approach to disturbance minimization for spacecraft utilizing controlled structures technology, MIT SERC Report \#2-90, MIT, June 1990.

[19] R. L. Fox And M. P. Kapoor, Rates of change of eigenvalues and eigenvectors, AIAA Journal, 6 (1968), pp. 2426-2429.

[20] G. F. Franklin and J. D. Powell, Digital Control of Dynamic Systems, Addison-Wesley Publishing Co., Inc., 1980.

[21] A. Gelb, ed., Applied Optimal Estimation, The M.I.T. Press, 1974 .

[22] J. J. Gilheany, Optimum selection of dampers for freely vibrating multidegree of freedom systems, Proceedings of Damping '89, II (1989), pp. FCC-1:18.

[23] C. Z. GREGORY, Reduction of large flexible spacecraft models using internal balancing theory, Journal of Guidance, Control, and Dynamics, 7 (1984), pp. 17-32.

[24] R. L. Grogan and R. A. LASKin, On multidisciplinary modeling of the space interferometry mission, in Proceedings of the American Control Conference, Philadelphia, PA, June 1998.

[25] H. L. Gutierrez, Performance Assessment and Enhancement of Precision Controlled Structures During Conceptual Design, PhD thesis, Massachusetts Institute of Technology, Department of Aeronautics and Astronautics, 1999.

[26] H. L. Gutierrez And D. W. Miller, Disturbance analysis of integrated structural/optical models with uncertainties, in Proceedings of the American Control Conference, Philadelphia, PA, June 1998 .

[27] T. K. Hasselman and J. D. Chrostowski, Evaluation of predictive accuracy in structural dynamic models, in Proceedings of the International Modal Analysis Conference, 1989, pp. 360366.

[28] T. K. Hasselman, J. D. Chrostowski, and T. J. Ross, Interval prediction in structural dynamic analysis, in Proceedings of the $33^{\text {rd }}$ AIAA/ASME/AHS/ASC Structures, Structural Dynamics, and Materials Conference, Dallas, TX, April 1992, pp. 1272-1284. AIAA Paper No. 92-2215.

[29] T. K. Hasselman, J. D. Chrostowski, and T. J. Ross, Propagation of Modeling Uncertainty Through Structural Dynamic Models, in Proceedings of the $35^{\text {th }}$ AIAA/ASME/AHS/ASC Structures, Structural Dynamics, and Materials Conference, Hilton Head, SC, April 1994.

[30] E. Hecht, Optics, Addison-Wesley Publishing Company, second ed., 1990.

[31] G. J. Hou AND G. Kogant, Sensitivity analysis of Lyapunov and Ricatti equations with application to controlsstructures integrated design, in Proceedings of the 34th AIAA/ASME/ASCE/AHS/ASC Structures, Structural Dynamics and Materials Conference, LaJolla, CA, April 1993 , pp. 1906-1915. AIAA Paper No. 93-1529.

[32] J. P. How, Robust Control Design with Real Parameter Uncertainty Using Absolute Stability Theory, PhD thesis, Massachusetts Institute of Technology, February 1993. SERC Report \#1-93.

[33] R. N. JACQUES, An approach to the preliminary design of controlled structures, Master's thesis, Massachusetts Institute of Technology, February 1991. SERC Report \#1-91.

[34] R. N. JACQUES, On-line System Identification and Control Design for Flexible Structures, PhD thesis, Massachusetts Institute of Technology, May 1995. SERC Report \#3-95.

[35] Jet Propulsion Laboratory, Origins Missions, URL <http://origins.jpl.nasa.gov/missions/sbobs.html>.

[36] Modeling and Analysis for Controlled Optical Systems User's Manual, 1997.

[37] Jet Propulsion Laboratory, Integrated Modeling of Optical Systems User's Manual V5.0, Jet Propulsion Laboratory, February 2000. JPL Publication 98-12, Rev. A.

[38] J. L. JUNKINS AND Y. KIM, Introduction to Dynamics and Control of Flexible Structures, AIAA Education Series, 1992.

[39] M. P. Kamat, Structural Optimization: Status and Promise, vol. 150 of Progress in Aeronautics and Astronautics, American Institute of Aeronautics and Astronautics, Washington, D.C., 1992.

[40] R. Kennedy, J. Turnage, and M. B. Jones, A meta-model for systems development through life cycle phases - coupling 
the isoperformance methodology with utility analysis, SAE, Aerospace Technology Conference and Exposition, Long Beach, CA, AIAA Technical Library (1990), p. 9.

[41] R. S. Kennedy, M. B. Jones, and D. R. Baltzley, Empirical demonstration of isoperformance methodology preparatory of an interactive expert computerized decision aid. final report, Tech. Rep. AIAA Technical Library, NTIS HC A03/MF A01, Essex Corporation, Orlando, FL, Feb 1988. AD-A202439 ARI-RN-88-93; Contract: MDA903-87-C-0603; Contract: DA PROJ. 2Q6-65502-M-770.

[42] R. S. Kennedy, M. B. Jones, and D. R. Baltzley, Optimal solutions for complex design problems: Using isoperformance software for human factors trade offs, In NASA. Lyndon B. Johnson Space Center, 2nd Annual Workshop on Space Operations Automation and Robotics (SOAR 1988), N89-19817 12-59 (1988), pp. 313-319.

[43] S. KENNY, Eigenvalue and eigenvector derivatives for structures, Final Report, MIT Course 2.093: Computer Methods in Dynamics, April 29,1997.

[44] R. A. LASKIN AND M. SAN MARTIN, Control/structure system design of a spaceborne optical interferometer, in Proceedings of the AAS/AIAA Astrodynamics Specialist Conference, Stowe, VT, August 1989. AAS Paper No. 89-424.

[45] A. LAUB, Computation of balancing transformations, Proceedings of JACC, 1 (1980).

[46] A. Laub, M. Heath, C. Paige, and R. Ward, Computation of system balancing transformations and other applications of simultaneous diagonalization algorithms, IEEE Trans. Automatic Control, (1987), pp. 115-122.

[47] P. Maghami, S. Gupta, K. B. Elliot, S. M. Joshi, And J. E. WALZ, Experimental validation of an integrated controlsstructures design methodology for a class of flexible space structures, in NASA Technical Paper 3462, November 1994.

[48] G. Mallory and D. W. Miller, Decentralized state estimation for flexible space structures, 40th AIAA Structural Dynamics and Materials Conference, (1999).

[49] G. J. Mallory, Development and Experimental Validation of Direct Controller Tuning for Spaceborne Telescopes, PhD thesis, Massachusetts Institute of Technology, Department of Aeronautics and Astronautics, April 2000. Report SERC 1-2000.

[50] B. Masters AND E. CRAWLEY, An experimental investigation of optimized precision optical controlled-structures, in Proceedings of the SPIE Conference on Smart Structures and Materials, vol. 3041, San Diego, CA, March 1997, pp. 348-359. Paper No. 3041-28.

[51] R. MASterson, Development and Validation of Empirical and Analytical Reaction Wheel Disturbance Models, Master's thesis, Massachusetts Institute of Technology, June 1999.

[52] J. W. MELODY, Discrete-frequency and broadband reaction wheel disturbance models, Interoffice Memorandum 3411-95200csi, Jet Propulsion Laboratory, June 1995.

[53] J. W. MELOdy AND G. W. NEAT, Integrated modeling methodology validation using the micro-precision interferom eter testbed: Assessment of closed-loop performance prediction capability, in Proceedings of the American Control Conference, Albuquerque, NM, June 1997.

[54] D. Miller, A. Curtis, And O. DE WeCK Et.Al., Architecting the Search for Terrestrial Planets and related Rrigins (astro), in SPIE International Symposium on Astronomical Telescopes and Instrumentation 2000, Munich, Germany, 2000.

[55] D. Miller, O. De WeCk, S. Uebelhart, R. Grogan, AND I. BASDOGAN, Integrated Dynamics and Controls Modeling for the Space Interferometry Mission (SIM), in IEEE Aerospace Conference, Big Sky, Montana, 2001.

[56] M. Milman, M. Salama, R. Scheid, and J. S. Gibson, Combined control-structural optimization, Computational Mechanics, (1991), pp. 1-18.

[57] B. C. MOORE, Principal component analysis of linear systems: Controllability, observability, and model reduction, IEEE Transactions on Automatic Control, AC-26 (1981), pp. 17-32.

[58] D. V. Murthy and R. T. HaftKa, Survey of methods for calculating sensitivity of general eigenproblems, in Sensitivity Analysis in Engineering, Langley Research Center, September 1986, pp. 177-196. NASA CP 2457.

[59] NASA, Origins: Roadmap for the Office of Space Science Ori- gins Theme, National Aeronautics and Space Administration, National Aeronautics and Space Administration, 1997.

[60] R. B. NELSON, Simplified calculations of eigenvector derivatives, AIAA Journal, 14 (1976), pp. 1201-1205.

[61] K. OGAtA, Modern Control Engineering, Prentice-Hall, Inc., 1990.

[62] M. C. O'Neal and J. T. Spanos, Optical pathlength control in the nanometer regime on the JPL phase $B$ interferometer testbed, in Proceedings of the SPIE Conference on Active and Adaptive Optical Systems, vol. 1542, San Diego, CA, July 1991, pp. 359-370.

[63] D. C. Redding and W. G. Breckenridge, Optical modeling for dynamics and control analysis, Journal of Guidance, Control, and Dynamics, 14 (1991), pp. 1021-1032.

[64] H. G. Rutten and M. A. van Venroois, Telescope Optics - A comprehensive Manual for Amateur Astronomers, WillmannBell Inc., Richmond, Virginia, fourth ed., 1999.

[65] Y. Sawaragi, H. Nakayama, and T. Tanino, Theory of Multiobjective Optimization, vol. 176 of Mathematics in Science and Engineering, Academic Press Inc., London, United Kingdom, i ed., 1985.

[66] R. E. Skelton, Model error concepts in control design, International Journal of Control, 49 (1989), pp. 1725-1753.

[67] G. Strang, Introduction to Linear Algebra, WellesleyCambridge Press, 1998

[68] S. A. Uebelhart, Conditioning, Reduction, and Disturbance Analysis of Large Order Integrated Models of Space-Based Telescopes, Master's thesis, Massachusetts Institute of Technology, February 2001.

[69] J. VAn DE Vegte, Feedback Control Systems, Prentice-Hall, Inc., 1990

[70] S. L. VENNERI, Future directions and needs in engineering tools, ICASE/LaRC/NSF/ARO Workshop on Computational Aerosciences in the 21st Century, (1998). Hampton, Virginia.

[71] P. H. Wirsching, T. L. PAez, And H. Ortiz, Random Vibrations: Theory and Practice, John Wiley \& Sons, Inc., 1995.

[72] K. Y.-L. Yang, Efficient Design of Robust Controllers for $\mathrm{H}_{2}$ Performance, PhD thesis, Massachusetts Institute of Technology, October 1996. SERC Report \#8-96.

[73] K. Zhou, J. C. Doyle, And K. Glover, Robust and Optimal Control, Prentice-Hall, Inc., 1996. 